\title{
Detection of Uniform and Nonuniform Differential Item Functioning by Item-Focused Trees
}

\author{
Moritz Berger \\ Gerhard Tutz \\ Ludwig-Maximilians-Universität München
}

\begin{abstract}
Detection of differential item functioning (DIF) by use of the logistic modeling approach has a long tradition. One big advantage of the approach is that it can be used to investigate nonuniform (NUDIF) as well as uniform DIF (UDIF). The classical approach allows one to detect DIF by distinguishing between multiple groups. We propose an alternative method that is a combination of recursive partitioning methods (or trees) and logistic regression methodology to detect UDIF and NUDIF in a nonparametric way. The output of the method are trees that visualize in a simple way the structure of DIF in an item showing which variables are interacting in which way when generating DIF. In addition, we consider a logistic regression method, in which DIF can be induced by a vector of covariates, which may include categorical but also continuous covariates. The methods are investigated in simulation studies and illustrated by two applications.
\end{abstract}

Keywords: logistic regression; differential item functioning; recursive partitioning; item-focused trees

\section{Introduction}

In recent years, differential item functioning (DIF) and DIF identification methods have been the areas of intensive research. DIF occurs if the probability of a correct response among persons with the same value of their underlying trait differs in subgroups, for example, if the difficulty of an item depends on the membership to a racial, ethnic, or gender subgroup. If a test contains DIF items, it may be unfair, that is, favor specific groups. When developing and using tests that measure latent abilities, one should be aware of the phenomenon of DIF. Ideally, tests should not contain suspicious items. If this cannot be obtained, one should at least know which items are DIF items and by which covariates DIF is generated. For more details on DIF, measurement bias, and possibly discrimination, see, for example, Holland and Wainer (1993), Millsap and Everson (1993), Osterlind and Everson (2009), Rogers (2005), and Zumbo (1999). 
A variety of methods to detect DIF have been proposed (for a more recent overview, see Magis, Bèland, Tuerlinckx, \& Boeck, 2010). One can in particular distinguish between item response theory (IRT) modeling approaches and test score methods (Magis, Tuerlinckx, \& De Boeck, 2015). The former assume that an IRT model holds in each group. Tests such as Lord's test or likelihood ratio (LR) tests are used to detect differences of item parameters between groups. IRT approaches have been used by Lord (1980), Raju (1988), and Holland and Wainer (1993), among other. Test score methods use a matching variable as, for example, Mantel-Haenszel test procedures (Holland \& Thayer, 1988) or logistic regression modeling (Swaminathan \& Rogers, 1990). We will use the logistic regression framework since it also allows us to investigate nonuniform DIF (NUDIF). Uniform DIF (UDIF) is present if individuals from different groups but with the same ability level have different probabilities in solving an item and those differences do not depend on the ability level. In NUDIF scenarios, the differences are not constant across ability levels, and the crossing item response curves may occur.

More recently, IRT-based DIF modeling has been extended to allow for continuous variables that induce DIF. The corresponding latent trait models contain many parameters since each item comes with an own vector of parameters. Therefore, maximum likelihood estimates are bound to fail. Tutz and Schauberger (2015) used a penalty approach to regularize parameter estimation, and Schauberger and Tutz (2015) used boosting techniques, whereas Tutz and Berger (2015) rely on recursive partitioning methods. A non-IRT modeling approach with regularization by penalties has been proposed by Magis, Tuerlinckx, and De Boeck (2015).

This article focuses on score-based methods. A recursive partitioning (tree based) method is proposed that allows for the identification of the items that carry DIF together with the variables that induce DIF. The variables can represent groups as in classical DIF detection techniques but can also include continuous variables like age. A strength of the method is that for continuous variables, it is not necessary to define a priori the intervals that are relevant; the method itself generates the intervals that are linked to DIF. The resulting tree visualizes in a simple way the structure of DIF in an item, showing which variables and interactions of variables generate DIF.

The method should be distinguished from the Rasch trees proposed by Strobl, Kopf, and Zeileis (2015). One difference between the methods is that Rasch trees are IRT-based methods designed for UDIF only. However, there are also strong differences between the methods for the detection of UDIF. By using tree methodology, the Rasch tree method also does not need prespecified subgroups and can handle continuous variables. Rasch trees recursively partition the covariate space to identify regions of the covariate space, in which DIF occurs by fitting separate item response models in these regions. Regions are suspected to be 


\section{Berger and Tutz}

relevant if the parameter estimates in the regions differ strongly. Therefore, regions in the covariate space are identified that show different difficulties, but the method does not flag items that are responsible. In contrast, the recursive partitioning method proposed here focuses on the detection of the items that are responsible for DIF. Recursive partitioning is used on the item level not on the global level, which treats all items simultaneously and therefore does not show which item is responsible for the occurrence of DIF. The method developed here is related to the recursive partitioning method proposed by Tutz and Berger (2015), which also flags DIF items. Tutz and Berger also give a more detailed discussion of the different ways of using tree methodology and illustrate the difference in applications.

In Section 2, we introduce the new recursive partitioning method based on the logistic regression approach for UDIF, and in Section 3, we present an illustrative example. A detailed description of the fitting procedure is given in Section 4. In Section 5, we consider the results of various simulations. Models for the extension to NUDIF are considered in Section 6. Finally, Section 7 contains two applications on real data.

\section{Logistic Regression Approaches to DIF}

In this section, basic logistic regression approaches to the detection of UDIF are described, and the alternative tree-based method is introduced.

\subsection{Linear Logistic Regression Approaches to DIF}

The basic test score-based method to detect UDIF was proposed by Swaminathan and Rogers (1990). It can be seen as a starting point of the method proposed here.

Let $Y_{p i} \in\{0,1\}, p=1, \ldots, P, i=1, \ldots, I$ denote the response when person $p$ tries to solve item $i$. Swaminathan and Rogers (1990) proposed to model the probability of solving an item as a function of the group membership and the test score by fitting the logistic regression model

$$
\log \left(\frac{P\left(Y_{p i}=1 \mid S_{p}, g\right)}{P\left(Y_{p i}=0 \mid S_{p}, g\right)}\right)=\eta_{p i}=\beta_{0 i}+S_{p} \beta_{i}+\gamma_{i g}
$$

where $g$ denotes the group, $S_{p}$ is the test score of person $p, \beta_{0 i}$ is the intercept, $\beta_{i}$ is the slope of item $i$, and $\gamma_{i g}$ are the group-specific parameters. In this model, the parameters $\beta_{01}, \ldots, \beta_{0 I}$ represent the item difficulties and the parameters $\beta_{1}, \ldots, \beta_{I}$ correspond to the discrimination parameters. Within this framework, the test scores are considered as proxies for the abilities of persons. For the detection of DIF, the most interesting parameters are the group-specific parameters $\gamma_{i 1}, \ldots, \gamma_{i G}$, where $G$ denotes the number of groups. They represent the DIF. In the simplest case of two groups, a reference group and a focal group, one 
chooses $\gamma_{i 1}=0$ for the reference group. Thus, for example, with groups defined by gender with female as the reference group, one has

$$
\beta_{0 i}+\gamma_{i, \text { male }} \text { for males and } \beta_{0 i} \text { for females. }
$$

If $\gamma_{i \text {,male }} \neq 0$, one has DIF in item $i$ generated by gender. The original framework for two groups was proposed by Swaminathan and Rogers (1990), and the extension to multiple groups was considered by Magis, Raîche, Béland, and Gérard (2011). In the multiple group, Case 1 of the $G$ groups, for example, the first group, has to be chosen as a reference group by setting $\gamma_{i 1}=0$.

DIF detection within the logistic regression framework typically uses LR statistics that test the null hypothesis $H_{0}: \gamma_{i 1}=\ldots=\gamma_{i G}=0$. If the hypothesis is rejected, Item $i$ is considered as a DIF item. Each item is tested separately at significance level $\alpha$ with the degrees of freedom equal to $G-1$, depending on the number of groups.

The basic concept can be simply extended to include continuous (and categorical) variables that might induce DIF. Let $x_{p}^{\top}=\left(x_{p 1}, \ldots, x_{p m}\right)$ be a vector of person-specific explanatory variables of length $m$. An extension of Model 1 for UDIF has the form

$$
\log \left(\frac{P\left(Y_{p i}=1 \mid S_{p}, \mathbf{x}_{p}\right)}{P\left(Y_{p i}=0 \mid S_{p}, \mathbf{x}_{p}\right)}\right)=\eta_{p i}=\beta_{0 i}+S_{p} \beta_{i}+\mathbf{x}_{p}^{\top} \boldsymbol{\gamma}_{i}
$$

The new intercept parameters in Model 3 are $\beta_{0 i}+x_{p}^{\top} \boldsymbol{\gamma}_{i}$, and they differ according to the characteristics of the person $\mathbf{x}_{p}$. The comparison of multiple groups is just a special case. Setting the first group as reference one defines the vector of explanatory variables $\mathbf{x}_{p}^{\top}=\left(x_{p 2}, \ldots, x_{p G}\right)$, where $x_{p g}=1$ if person $p$ is from group $g$ and 0 otherwise. The corresponding vector of parameters for 1 item $i$ is $\boldsymbol{\gamma}_{i}^{\top}=\left(\gamma_{i 2}, \ldots, \gamma_{i G}\right)$. UDIF is present in this item if $\boldsymbol{\gamma}_{i} \neq \mathbf{0}$. To investigate DIF, one uses a global test for the whole parameter vector, $H_{0}: \boldsymbol{\gamma}_{i}=0$. The alternative hypothesis is that at least one of the parameters is unequal to zero. The hypotheses are tested separately for each item at significance level $\alpha$. Due to the design of the tests, the approach identifies the items that carry DIF but does not contain any information about the components of $\mathbf{x}_{p}$ that are responsible for DIF. Although being a straightforward extension of the fixed groups DIF Model 1, the extension (3) seems not to have been investigated so far.

We will refer to the multiple groups Model 1 as the classical logistic regression modeling approach and to Model 3 as the extended approach. It should be mentioned that the extended approach (including continuous or categorical covariates) is already implicitly contained in the approach proposed by Magis et al. (2011). The approach of Magis et al. (2015) provides an extra layer of complexity with penalization on the DIF parameters. The main contribution in the present article, which is outlined in the following sections, is that the linear part of the basic model is replaced by tree structured fitting. 


\subsection{A Tree Representation of DIF}

DIF detection based on the logistic regression model as described in the previous section has some limitations and drawbacks. If one uses the traditional version with $G$ groups, DIF can be induced only by group membership. A continuous variable like age has to be divided into intervals to obtain groups without knowing which intervals are important. The extended version with a linear predictor is restricted by the assumption that the DIF effect is linear. Moreover, the tests that are used to identify items that carry DIF do not show which variables are responsible for DIF, at least not in a simple way. The proposed recursive partitioning method avoids the problem that reference and focal groups have to be specified a priori. By recursive splitting, the method itself identifies the groups that induce DIF if they are present.

The general concept of recursive partitioning has its roots in automatic interaction detection. The most popular modern version is due to Breiman, Friedman, Olshen, and Stone (1984) and is known by the name classification and regression trees. An alternative approach is the recursive partitioning framework based on conditional inference proposed by Hothorn, Hornik, and Zeileis (2006). The basic method is conceptually very simple. By binary recursive partitioning, the feature space is partitioned into a set of rectangles, and on each rectangle, a simple model (e.g., a constant) is fitted. An easily accessible introduction into basic concepts is found in Hastie, Tibshirani, and Friedman (2009); an overview with a focus on psychometrics was given by Strobl, Malley, and Tutz (2009). It should be noted that the method proposed here is based on the same idea, but there is one crucial difference. When fitting a model, we do not fit two separate models within the rectangles obtained by partitioning. We fit one closed model and only the intercept is partitioned into rectangles. This yields item-focused trees (IFT) in contrast to global trees as used by conventional Rasch trees.

Building a tree means to successively find a partition of the predictor space, where each node represents a subset of the predictor space. The terminal nodes of the tree build a disjoint partition of the predictor space and correspond to the relevant subregions of interest. When growing a tree, one typically splits one node $A$ into two subsets $A_{1}$ and $A_{2}$. The split is determined by exactly one variable and the construction of the split depends on the scale of the variable. In the following considerations, we will focus on metrically scaled and ordinal variables. In this case, the partition into two subsets has the form

$$
A_{1}=A \cap\left\{x_{j} \leq c\right\} \quad \text { and } \quad A_{2}=A \cap\left\{x_{j}>c\right\},
$$

with regard to threshold $c$ on variable $x_{j}$. Given the covariates $\mathbf{x}_{p}$, one can account for UDIF by building a partition of the respondents with differing intercepts. The first split with regard to the $j$ th variable and corresponding split point $c_{j}$ means to fit the model with predictor

$$
\eta_{p i}=S_{p} \beta_{i}+\left[\gamma_{i l}^{[1]} I\left(x_{p j} \leq c_{j}\right)+\gamma_{i r}^{[1]} I\left(x_{p j}>c_{j}\right)\right],
$$


where $I(\cdot)$ denotes the indicator function with $I(a)=1$ if $a$ is true and $I(a)=0$ otherwise. The parameter $\gamma_{i l}^{[1]}$ denotes the intercept in the left node $\left(x_{p j} \leq c_{j}\right)$ and $\gamma_{i r}^{[1]}$ the intercept in the right node $\left(x_{p j}>c_{j}\right)$. For example, one split with regard to the binary covariate gender yields the intercepts

$$
\gamma_{i l}^{[1]}=\gamma_{i, \text { male }} \text { for males and } \gamma_{i r}^{[1]}=\gamma_{i \text {,emale }} \text { for females. }
$$

This parametrization is an equivalent representation of (2). The main difference is that the two subgroups of interest are not predefined but determined by a split in variable $j$ at split-point $c_{j}$. To determine the first split, one examines all the null hypotheses $H_{0}: \gamma_{i l}^{[1]}=\gamma_{i r}^{[1]}$. If $H_{0}$ cannot be rejected for any combination of variable and split point, the item is considered to be free of DIF. In the proposed algorithm, LR tests are used to examine the null hypotheses. In the very first step, one chooses the combination of item, variable, and split point with the smallest $p$ value of the corresponding test. If a significant effect is found, the first split into left and right node is carried out for the selected item. In Section 1, the splitting criterion is described in more detail.

One further split, for example, in the right node $\left(x_{p j}>c_{j}\right)$, with regard to the $s$ th variable at split point, $c_{s}$ yields the two daughter nodes $I\left(x_{p j}>c_{j}\right) I\left(x_{p s} \leq c_{s}\right)$ and $I\left(x_{p j}>c_{j}\right) I\left(x_{p s}>c_{s}\right)$. The new nodes are both defined by the product of two indicator functions. In general, each node can be represented by a product of several indicator functions, namely,

$$
\operatorname{node}\left(\mathbf{x}_{p}\right)=\prod_{b=1}^{B} I\left(x_{p j_{b}}>c_{j_{b}}\right)^{a_{b}} I\left(x_{p j_{b}} \leq c_{j_{b}}\right)^{1-a_{b}},
$$

where $B$ is the total number of indicator functions or branches, $c_{j_{b}}$ is the selected split point in variable $j_{b}$, and $a_{b} \in\{0,1\}$ indicates which of the indicator functions, below or above the threshold, is involved. The resulting predictor of the model for Item $i$ after several splits with terminal nodes $\ell=1, \ldots, L_{i}$ is then given by

$$
\eta_{p i}=S_{p} \beta_{i}+\sum_{\ell=1}^{L_{i}} \gamma_{i \ell} \operatorname{node}_{i \ell}\left(\mathbf{x}_{p}\right)=S_{p} \beta_{i}+\operatorname{tr}_{i}\left(\mathbf{x}_{p}\right),
$$

where $\operatorname{tr}_{i}\left(\mathbf{x}_{p}\right)$ is the tree component containing subgroup-specific intercepts represented by the terminal nodes $\operatorname{node}_{\ell}\left(\mathbf{x}_{p}\right)$. The proposed algorithm yields an individual tree for each item that was selected to carry DIF. If an item is never chosen for splitting, it is assumed to be free of DIF, and the fitted "tree" is a constant $t r_{i}\left(\mathbf{x}_{p}\right)=\beta_{0 i}$.

We use the abbreviation IFT for item-focused trees based on the logistic regression framework. 

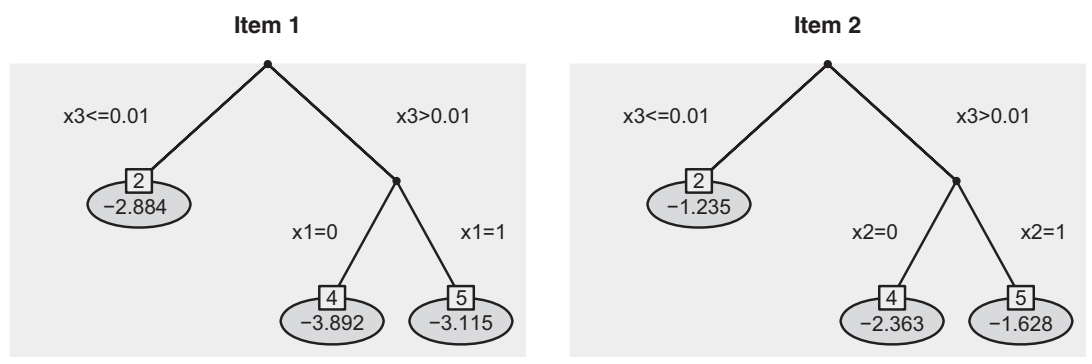

FIGURE 1. Estimated trees of Items 1 and 2 for the illustrative example. Estimated coefficients $\gamma_{i \ell}$ are given in each leaf of the trees.

\section{An Illustrative Example}

The procedure is now first illustrated by the use of artificial data. We consider data $Y_{p i}, p=1, \ldots, 800, i=1, \ldots, 20$ that are generated by a two-parameter model (2PL) with DIF. The basic 2PL model has the form

$$
P\left(Y_{p i}=1 \mid \theta_{p}, b_{i}, a_{i}\right)=\frac{\exp \left(a_{i}\left(\theta_{p}-b_{i}\right)\right)}{1+\exp \left(a_{i}\left(\theta_{p}-b_{i}\right)\right)},
$$

where $\theta_{p}$ denotes the person ability, $b_{i}$ the item difficulty, and $a_{i}$ the item discrimination. We first generate person parameters $\theta_{p}$ and item difficulties $b_{i}$ from a standard normal distribution and item discriminations $a_{i}$ from a uniform distribution. However, instead of generating data from the 2PL model, we assume that the difficulties of 2 of the 20 items depend on covariates in a complex pattern.

In detail, we consider three covariates, two binary variables $x 1, x 2 \sim B(1,0.5)$ and one standard normal distributed variable $x_{3} \sim N(0,1)$. In Item 1 , DIF is induced by $x_{1}$ and $x_{3}$, and the modified value of the difficulty is determined by the step functions $b_{1, \bmod }=b_{1}+0.8 \cdot I\left(x_{3}>0\right)+0.8 \cdot I\left(\left\{x_{3}>0\right\} \cap\left\{x_{1}=0\right\}\right)$; in Item 2, DIF is induced by $x_{2}$ and $x_{3}$, and we use the step functions $b_{2 \text {, mod }}=b_{2}+0.8 \cdot I\left(x_{3}>0\right)+0.8 \cdot I\left(\left\{x_{3}>0\right\} \cap\left\{x_{2}=0\right\}\right)$, which represents an interaction between variables $x_{2}$ and $x_{3}$. In order to evaluate the fitting procedure, 100 data sets were generated.

Figure 1 shows one exemplary estimation result of the 2 items with DIF (Items 1 and 2) when fitting IFT. The estimation in this example is quite perfect because the true underlying tree structure is detected for both items and no further item is falsely identified as DIF item. It can be seen from the trees that there are three groups represented by three terminal nodes, respectively. For Item 1, it is distinguished between $\left\{x_{3} \leq 0.01\right\}$ and $\left\{x_{3}>0.01\right\}$, and within this group between $\left\{x_{1}=0\right\}$ and $\left\{x_{1}=1\right\}$. The corresponding intercepts $\hat{\gamma}_{1 \ell}$ and $\hat{\gamma}_{2 \ell}, l=1, \ldots, 3$, 
of the estimated Model 5 are given in each leaf of the trees. According to Model 5 , the probability to solve the item correctly increases with increasing intercepts. From the estimates in Figure 1, one can derive that Item 1 is most difficult for region $\left\{x_{3}>0.01\right\} \cap\left\{x_{1}=0\right\}$ and Item 2 is most difficult for $\left\{x_{3}>0.01\right\} \cap\left\{x_{2}=0\right\}$. These results are exactly in line with the true simulated effects. In the simulations in Section 1, these artificial data are, inter alia, again considered in more detail.

\section{Fitting Procedure}

In this section, we give details about the fitting procedure for our proposed IFT to investigate UDIF.

\subsection{Concepts}

When building trees for single items in each step, one has to identify the best split due to an optimality criterion and decide if there is a relevance to perform the split or not. The second determines when to stop and therefore at the same time determines the size of the trees.

Since the approach is based on logistic regression models, it is quite natural to use test-based splits. In each step of the fitting procedure, one obtains $p$ values for the two parameters that are involved in the splitting. In our previous notation, one examines all the null hypotheses $H_{0}: \gamma_{i l}=\gamma_{i r}$ for each combination of item, variable, and split point. One simply selects the combination as the optimal one that has the smallest $p$ value. As a test statistic, we use the LR test statistic. Computing the LR test statistic requires us to estimate both models, the full model and the restricted model under $H_{0}$. We nevertheless prefer the LR statistic because it corresponds to selecting the model with minimal deviance. This criterion on the other hand is equivalent to minimizing the entropy, which belongs to the family of impurity measures that were already introduced as splitting criteria by Breiman et al. (1984).

In order to decide whether the split should be performed or not, we use a concept based on maximally selected statistics. The idea is to perform a test that investigates the null hypotheses of independence of the response and one of the covariates at the global variable level. For one fixed item $i$ and variable $j$, one simultaneously considers all LR test statistics $T_{j c_{j}}$, where $c_{j}$ are from the set of possible split points and computes the maximal value statistic $T_{j}=\max _{c_{j}} T_{j c_{j}}$. The $p$ value that can be obtained by the distribution of $T_{j}$ provides a measure for the relevance of variable $j$. The result is not influenced by the number of split points, since it has already taken into account (see Hothorn \& Lausen, 2003; Shih, 2004; Shih \& Tsai, 2004; Strobl, Boulesteix, \& Augustin, 2007). As the distribution of $T_{j}$ in general is unknown, we use a permutation test to obtain a decision on the null hypothesis. The distribution of $T_{j}$ is determined by computing the maximal 
value statistics based on random permutations of variable $j$. A random permutation of variable $j$ breaks the relation of the covariate and the response in the original data. By computing the maximal value statistics for a large number of permutations, one obtains an approximation of the distribution under the null hypothesis and a corresponding $p$ value. All computations in the present article are based on 1,000 permutations. Given overall significance level $\alpha$, the local significance level of one permutation test for fixed item and variable is chosen as $\alpha / m$. Using this adaption, the probability for each item without DIF of being falsely classified as DIF item is controlled by $\alpha$. As usual in DIF detection, one controls for the Type I error that is also known as false alarm rate. However, on the item level, one should adapt for multiple testing. Choosing $\alpha / m$ ensures that the probability of falsely identifying at least one variable as responsible for DIF is controlled by $\alpha$.

It should be noted that, in general, the number of permutations should depend on the number of covariates $m$. In our simulations and applications, the maximal number of covariates is 3 . Therefore, with a sample of 1,000 permutations, the $p$ values are determined with sufficient accuracy. From our experience, it is recommended to use at least 200 permutations for settings with one covariate and to increase the number of permutations by 200 per covariate. Thus, lower bound for settings with three covariates are 600 permutations.

\subsection{The Basic Algorithm}

The basic algorithm for UDIF is the following.

\section{Basic Algorithm-UDIF}

Step 1 (Initialization)

Set counter $v=1$

(a) Estimation

For all items $i=1, \ldots, I$, fit all the candidate logistic models with predictor

$$
\begin{gathered}
\eta_{p i}=S_{p} \beta_{i}+\gamma_{i 1} I\left(x_{p j} \leq c_{i j k}\right)+\gamma_{i 2} I\left(x_{p j}>c_{i j k}\right), \\
j=1, \ldots, m, \quad k=1, \ldots, K_{j} .
\end{gathered}
$$

(b) Selection

Select the model that has the best fit. Let $c_{i_{1}, j_{1}, k_{1}}$ denote the best split, which is found for item $i_{1}$ and variable $x_{j_{1}}$. 
(c) Splitting decision

Select the item and variable with the largest value of $T_{j}$. Carry out permutation test for this combination with significance level $\alpha / m$. If significant, fit the selected model yielding estimates $\hat{\beta}_{i}, \hat{\gamma}_{i_{1}, 1}, \hat{\gamma}_{i_{1}, 2}$ and nodes node $i_{i_{1}, 1}$, node $_{i_{1}, 2}$, set $v=2$. If not, stop, no DIF detected.

Step 2 (Iteration)

(a) Estimation:

For all items $i=1, \ldots, I$ and already built nodes $\ell=1, \ldots, L_{i v}$, fit all the candidate logistic models with new intercepts

$$
\gamma_{i, L_{i v}+1} \operatorname{node}_{i \ell} I\left(x_{p j} \leq c_{i j k}\right)+\gamma_{i, L_{i v}+2} \operatorname{node}_{\ell l} I\left(x_{p j}>c_{i j k}\right)
$$

for all $j$ and remaining, possible split points $c_{i j k}$.

(b) Selection

Select the model that has the best fit yielding the split point $c_{i_{v}, j_{v}, k_{v}}$, which is found for item $i_{v}$ in node $i_{v}, \ell v$ and variable $x_{j_{v}}$.

(c) Splitting decision

Select the node and variable with the largest value of $T_{j}$. Carry out permutation test for this combination with significance level $\alpha / m$. If significant, fit the selected model yielding the additional estimates $\hat{\gamma}_{i_{v}, L_{i v, v}+1}, \hat{\gamma}_{i_{v}, L_{i v}, v}+2$, set $v=v+1$. If not, stop.

\section{Simulations}

In the following, we consider data $Y_{p i}, p=1, \ldots, P, i=1, \ldots, I$ that are generated according to the 2PL, which is a dichotomous IRT model of the form

$$
P\left(Y_{p i}=1 \mid \theta_{p}, a_{i}, b_{i}\right)=\frac{\exp \left(a_{i}\left(\theta_{p}-b_{i}\right)\right)}{1+\exp \left(a_{i}\left(\theta_{p}-b_{i}\right)\right)},
$$

where $\theta_{p}$ are the person abilities, $b_{i}$ are the item difficulties, and $a_{i}$ are the item discrimination parameters.

We consider several simulation scenarios where in a first step, the person parameters $\theta_{p}$ and the item difficulties $b_{i}$ are independently drawn from a standard normal distribution and the item discrimination parameters $a_{i}$ are uniformly distributed, $a_{i} \sim U(0,1)$. If an Item $i$ is assumed to show UDIF, the corresponding parameter $b_{i}$ is subsequently transformed by specific step functions in each scenario. A detailed description is given in the respective section. 


\section{Berger and Tutz}

In each simulation scenario, we vary the number of persons, $P \in\{400,800\}$, the number of items, $I \in\{20,40\}$, and the percentage of DIF items, which is $0 \%, 10 \%$, or $20 \%$. In the cases with DIF, we additionally consider three different strengths of DIF, defined by a constant $c \in$ $\{0.4,0.8,1.6\}$ for the simulations with UDIF and $c \in\{0.3,0.6\}$ for the simulations with NUDIF. More details are given in the respective sections. In total, this results in 28 different settings (4 without DIF and 24 with DIF), respectively. In each setting, 100 data sets were generated. During estimation, each permutation test is based on 1,000 permutations.

In order to evaluate the performance of the proposed tree-based Model 5, we compute true positive rates (TPR), also named hit rates, and false positive rates (FPR), which correspond to the Type I error rates if no DIF is present. We distinguish between TPR and FPR on the item level and for the combination of item and variable. Let each item be characterized by a vector $\boldsymbol{\delta}_{i}^{T}=\left(\delta_{i 1}, \ldots, \delta_{i m}\right)$, where $m$ denotes the number of covariates, with $\delta_{i j}=1$ if item $i$ has DIF in variable $j$ and $\delta_{i j}=0$ otherwise. An item is a non-DIF item if $\boldsymbol{\delta}_{i}^{T}=(0, \ldots, 0)$; if one of the components is 1 , it is a DIF item. With indicator function $I(\cdot)$, the criteria to judge the identification of items with DIF are:

- TPR on the item level:

$$
\mathrm{TPR}_{I}=\frac{1}{\#\left\{i: \boldsymbol{\delta}_{i} \neq \mathbf{0}\right\}} \sum_{i: \delta_{i} \neq \mathbf{0}} I\left(\hat{\boldsymbol{\delta}}_{i} \neq \mathbf{0}\right) .
$$

- FPR on the item level:

$$
\mathrm{FPR}_{I}=\frac{1}{\#\left\{i: \delta_{i}=\mathbf{0}\right\}} \sum_{i: \delta_{i}=\mathbf{0}} I\left(\hat{\boldsymbol{\delta}}_{i} \neq \mathbf{0}\right) .
$$

- TPR for the combination of item and variable:

$$
\mathrm{TPR}_{I V}=\frac{1}{\#\left\{i, j: \delta_{i j} \neq 0\right\}} \sum_{i, j: \delta_{i j} \neq 0} I\left(\hat{\delta}_{i j} \neq 0\right) .
$$

- FPR for the combination of item and variable:

$$
\mathrm{FPR}_{I V}=\frac{1}{\#\left\{i, j: \delta_{i j}=0\right\}} \sum_{i, j: \delta_{i j}=0} I\left(\hat{\delta}_{i j} \neq 0\right) .
$$

The methods that are considered in the simulations are:

- Logistic which denotes the classical regression method proposed by Swaminathan and Roges (1990) and Magis et al. (2011). If the predictor is a vector with possibly continuous variables, it denotes the extended logistic model.

- IFT based on the logistic model which describes the recursive partitioning method proposed here. 

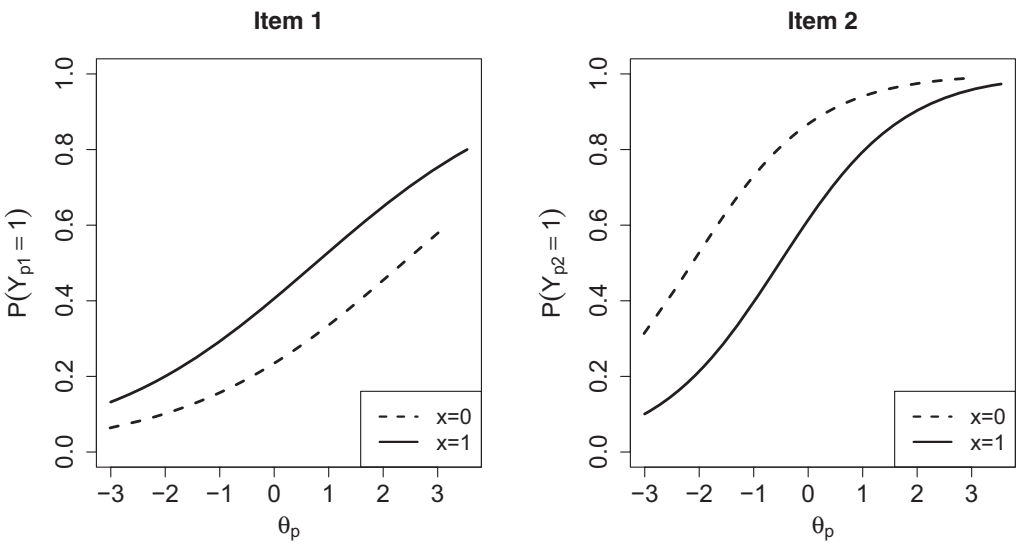

FIGURE 2. Item characteristic curves of Items 1 and 2 for one setting in the simulation with one binary predictor.

\subsection{Results}

First we consider data with two or more groups defined by one covariate. The main objective here is to compare the proposed IFT approach to the classical logistic approach, which is well established for the comparison of multiple groups. Later, we give detailed results of the proposed IFT, considering more complex data constellations with several predictors.

5.1.1. One binary predictor. We start with one binary covariate $x \in\{0,1\}$. In this simple case, the investigations reduce to the comparison of two groups. UDIF is present if the item difficulties $b_{i}$ differ between the two groups. The difference is simulated by $b_{i, \bmod }=b_{i}+c \cdot I(x=0)$ for one half of the DIF items and $b_{i, \bmod }=$ $b_{i}+c \cdot I(x=1)$ for the other half of the DIF items. The strength of DIF is determined by the constant $c \in\{0.4,0.8,1.6\}$. A difference in difficulties of 0.4 is very small, whereas a difference of 1.6 between the two groups is quite large. DIF is generated symmetrically because one half of DIF items favor the first group $(x=1)$ and the other DIF items favor the second group $(x=0)$. For illustration, Figure 2 shows the item characteristic curves (ICC) of the 2 items with DIF for the setting with $P=800, I=20,10 \%$ DIF items, and $c=1.6$. From the probabilities, it can be seen that Item 1 is more difficult for $x=0$ and Item 2 is more difficult for $x=1$. The item locations (value of $\theta_{p}$ with probability 0.5 ) differ between the two groups, but the item discriminations (steepness at the item location) are the same for both groups.

For the comparison of the results, we use receiver operating characteristic (ROC) curves, which have also been used by Magis et al. (2015) and Schauberger and Tutz (2015) to evaluate the performance of DIF detection methods. TPRs and 


$$
P=400, I=40,20 \% \text { DIF }
$$
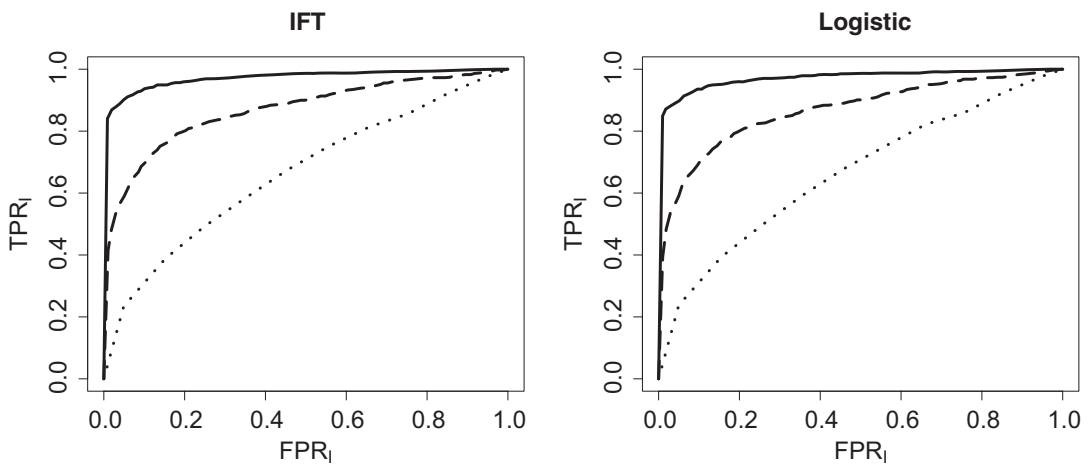

DIF strength $\mathrm{c}=0.8$

IFT
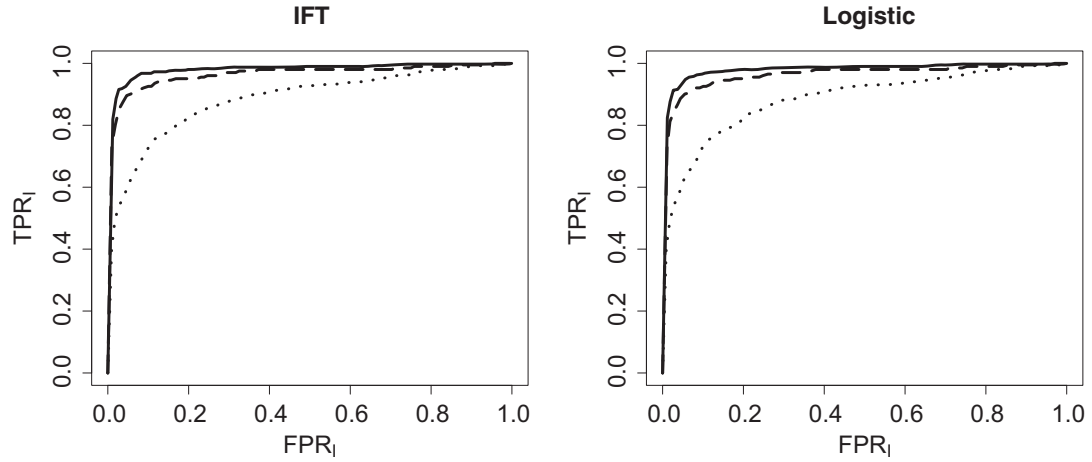

FIGURE 3. Average receiver operating characteristic curves for six settings in the simulation with one binary predictor. The upper panel shows the curves for three settings with fixed components and varying differential item functioning (DIF) strength (different line types) and the lower panel shows the curves for three settings with the same DIF strength.

FPRs on the item level were computed for increasing significance level $\alpha \in[0,1]$. The corresponding ROC curve is then obtained by plotting $\left(\mathrm{FPR}_{I}, \mathrm{TPR}_{I}\right)$ as a function of $\alpha$. Figure 3 shows the ROC curves for six of 24 settings with DIF as the average over 100 repetitions, respectively. The upper panels show the settings with $P=400 ; I=40 ; 20 \%$ DIF; and varying DIF strength $c=1.6$ (solid line), $c=0.8$ (dashed line), and $c=0.4$ (dotted line). The lower panels show settings with the same DIF strength $c=0.8$ and $P=800$, $I=20,20 \%$ DIF (solid line); $P=800, I=20,10 \%$ DIF (dashed line); and $P=400, I=40,10 \%$ DIF (dotted line). The resulting curves for IFT are given in the left panel, and the resulting curves for the classical logistic method are given in the right panel. From Figure 3, it can be seen that the DIF strength (value 
TABLE 1.

Average FPR on the Item Level at Significance Level $\alpha=.05$ for the Four Settings Without Differential Item Functioning in the Simulation With One Binary Predictor

\begin{tabular}{lccccc}
\hline & \multicolumn{2}{c}{$I=20$} & & \multicolumn{2}{c}{$I=40$} \\
\cline { 2 - 3 } \cline { 5 - 6 } & $P=400$ & $P=800$ & & $P=400$ & $P=800$ \\
\hline IFT & .050 & .051 & & .049 & .050 \\
Logistic & .052 & .048 & & .051 & .050 \\
\hline
\end{tabular}

Note. $\mathrm{FPR}=$ false positive rate; IFT $=$ item-focused trees.

of $c$ ) and the sample size $P$ have a strong effect on the detection performance, whereas the percentage of DIF items does not have a strong impact.

Although the global performance strongly varies over the different settings, there are only minor differences between the two methods as far as their performance is concerned. All settings we considered, not only the one presented in Figure 3, showed nearly no differences between the two methods. This result is not really surprising. After one split in to the binary predictor $x$, the obtained Model 5 for 1 item is exactly the same as Model 3, which is used for testing when using the classical logistic approach. In this case, the only remaining difference is the use of different test statistics to obtain a decision. Nevertheless, the classical and the new approach obviously show the same performance. This is important because the tree-based approach, which can also be used in more complex settings with many variables, can also be used in the case of two groups without loss of efficiency.

The construction of ROC curves is an efficient tool but is informative only if DIF is present. Therefore, we separately consider the case without DIF. The average FPRs with significance level $\alpha=.05$ for the four settings without DIF are given in Table 1. The absence of DIF is a baseline situation to check a possible inflation of FPRs. According to the obtained results, this is not the case. The IFT approach (approximately) holds the significance level as does the classical logistic approach. Again, the two approaches nearly yield the same results.

5.1.2. One ordered predictor. Here, we consider an ordered factor $x \in$ $\{1, \ldots, 6\}$. The difference in item difficulties is simulated by $b_{i, \text { mod }}=b_{i}+c$. $I(x>3)$ for one half of DIF items and $b_{i, \bmod }=b_{i}+c \cdot I(x \leq 3)$ for the other half of DIF items. Hence, there are only two groups that show a true difference, respectively. All the other specifications remain the same as in the previous Section 5.1.1. The ROC curves of six selected examples are given in Figure 4. The chosen settings are the same as in Figure 3. The left panel now refers to the settings with varying DIF strength and fixed $I, P$, and percentage of DIF items. The right panel refers to the three settings with constant DIF strength. 

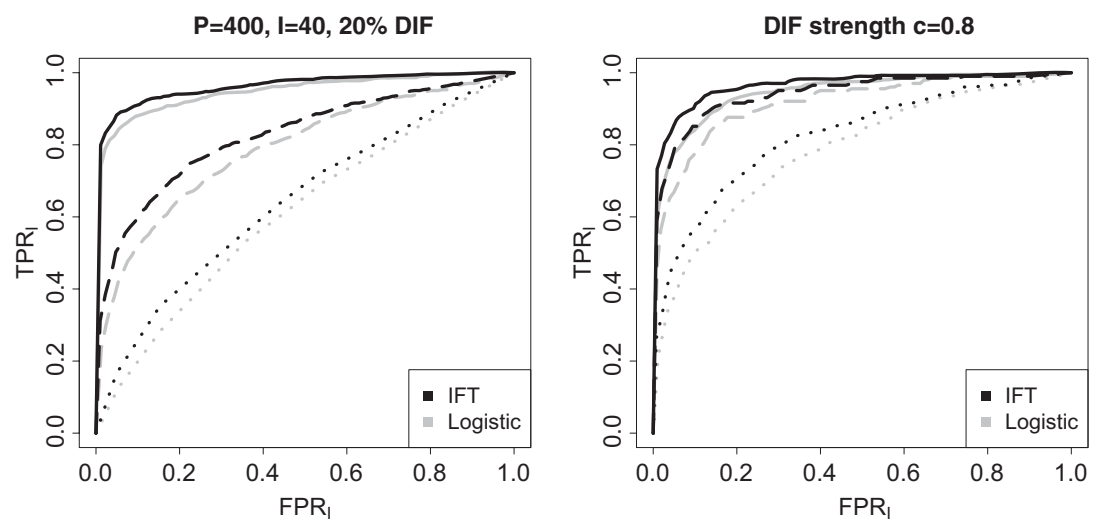

FIGURE 4. Average receiver operating characteristic curves for six settings in the simulation with one ordered predictor. The left panel shows the curves for three settings with fixed components and varying differential item functioning (DIF) strength (different line types) and the right panel shows the curves for three settings with the same DIF strength.

In contrast to the comparison of two groups, now there are visible differences between the performances of the two methods. The ROC curves show that IFT (black lines) outperforms the classical logistic (gray lines) across the whole range of $\alpha$. The ROC curves of the new approach are everywhere above the ROC curves of the classical approach. These findings are consistent throughout all settings. The differences are strongest for the settings with medium DIF $(c=0.8)$. The reason for the better performance of IFT is that it is able to use the ordering of the categories. Since DIF is linked to the ordinal scale of the factor, a method that is able to exploit the ordering should perform better than the classical method that just distinguishes between the groups. It is noteworthy that in Figure 4, the performance of the settings with a large number of persons and medium DIF strength (solid and dashed line in the right panel) is fairly similar to the performance with a small number of persons and strong DIF (solid line in the left panel). This underlines that an increase of sample size strongly contributes to improve the detection performance.

5.1.3. Several predictors. In the following simulations, we consider three covariates, two binary variables $x_{1}, x_{2} \sim B(1,0.5)$, and one standard normal distributed variable $x_{3} \sim N(0,1)$. Since IFT allows for determining the variables that are responsible for DIF, true positive and FPRs for the combination of item and variable can be computed. In the following, all the presented results are based on computations with significance level $\alpha=.05$. To account for the three covariates in the model, the local significance level for one permutation test is $.05 / 3$.

Before simulating items with DIF, we first investigate the baseline situation without DIF. The average FPRs for the four settings (varying number of 
TABLE 2.

Average FPR at Significance Level $\alpha=.05$ for the Four Settings Without DIF in the Simulation With Three Covariates.

\begin{tabular}{ccccccc}
\hline & \multicolumn{2}{c}{$I=20$} & & \multicolumn{2}{c}{$I=40$} \\
\cline { 3 - 3 } \cline { 5 - 6 } & & $P=400$ & $P=800$ & & $P=400$ & $P=800$ \\
\hline \multirow{2}{*}{ IFT } & $\mathrm{FPR}_{I}$ & .027 & .021 & & .024 & .022 \\
& $\mathrm{FPR}_{I V}$ & .009 & .007 & & .008 & .007 \\
\hline
\end{tabular}

Note. $\mathrm{FPR}=$ false positive rate DIF $=$ differential item functioning; $\mathrm{IFT}=$ item-focused trees.

persons and items) without DIF are given in Table 2. It is seen that IFT yields small FPRs. The procedure is conservative and does not fully use the specified significance level. On average, only 1 item is misleadingly identified as DIF item. FPRs for the combination of item and variable are much smaller. With 40 items, the value 0.008 means that only one split with regard to a variable that was not inducing DIF was falsely executed during estimation.

DIF in the First Variable. In the settings with DIF, first DIF is simulated as in the simulation with one binary predictor only (Section 5.1.1). If DIF is present, the item difficulties $b_{i}$ differ between the two groups defined by the binary covariate $x_{1}$. Hence, the underlying true model is defined by one split in $x_{1}$. Boxplots of true positive and FPRs of the 24 settings with DIF are given in Figure 5. The results on the item level are in light gray and are given on the left of each panel, and the results for the combination of item and variable are in dark gray and are given on the right of each panel. In addition, the significance level $\alpha=.05$ is marked as a reference by dashed lines. It is seen from Figure 5 that IFT shows good overall performance for medium and strong DIF, in particular if the number of persons is large. For small DIF effects, the number of persons definitely has to be large. TPRs are high in the settings with $P=800$ and $c=1.6$. Here, a clear separation between DIF and non-DIF items is seen. For the setting in the lower left of Figure 5 with $P=400, I=40,20 \%$ DIF items, and $c=1.6$, one observes a TPR of 0.5 in 68 of the 100 data sets, and therefore the box reduces to one value. In the settings with small DIF $(c=0.4)$ and a small number of persons $(P=400)$, the method is hardly able to detect the corresponding items. However, as is seen from Figure 4, alternative methods also show poor performance if DIF is weak. FPRs are very small throughout all settings, in particular the global significance level holds (with a tendency of the method to be conservative). It is noteworthy that the TPRs for the combination of item and variable in all settings are very similar to the TPRs for items. Therefore, IFT is able to simultaneously identify the items and variables that are responsible for DIF. Similar pictures resulted if the covariates $x_{1}, x_{2}$, and $x_{3}$ were correlated with medium-sized 


\section{Berger and Tutz}

$10 \%$ DIF

\section{TPR/FPR}
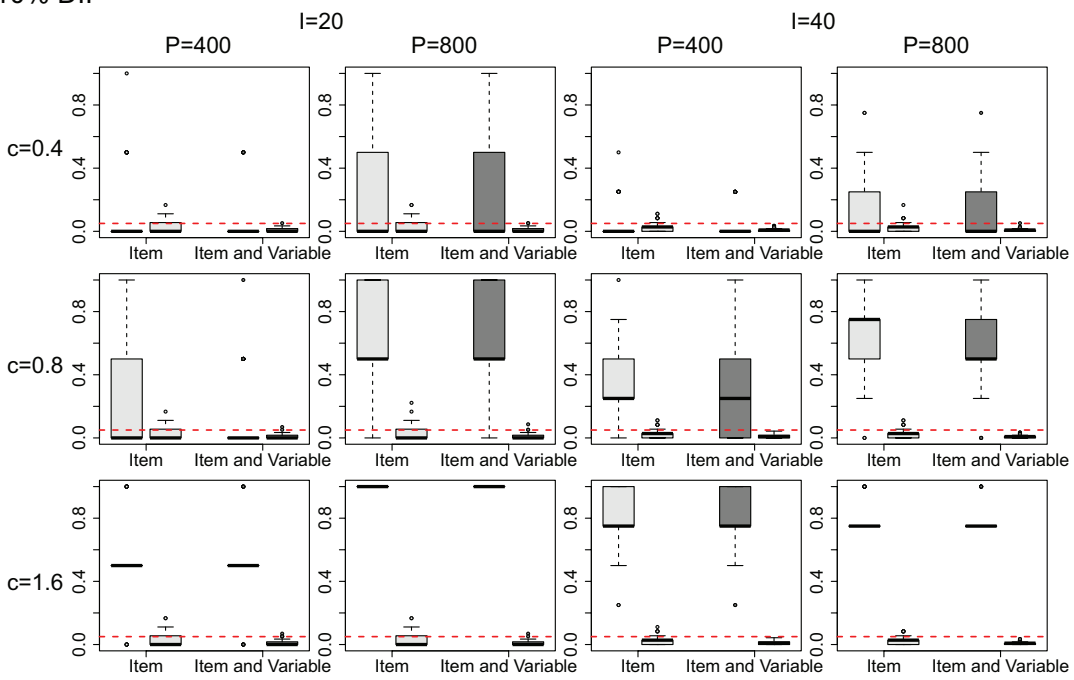

$20 \%$ DIF

TPR/FPR

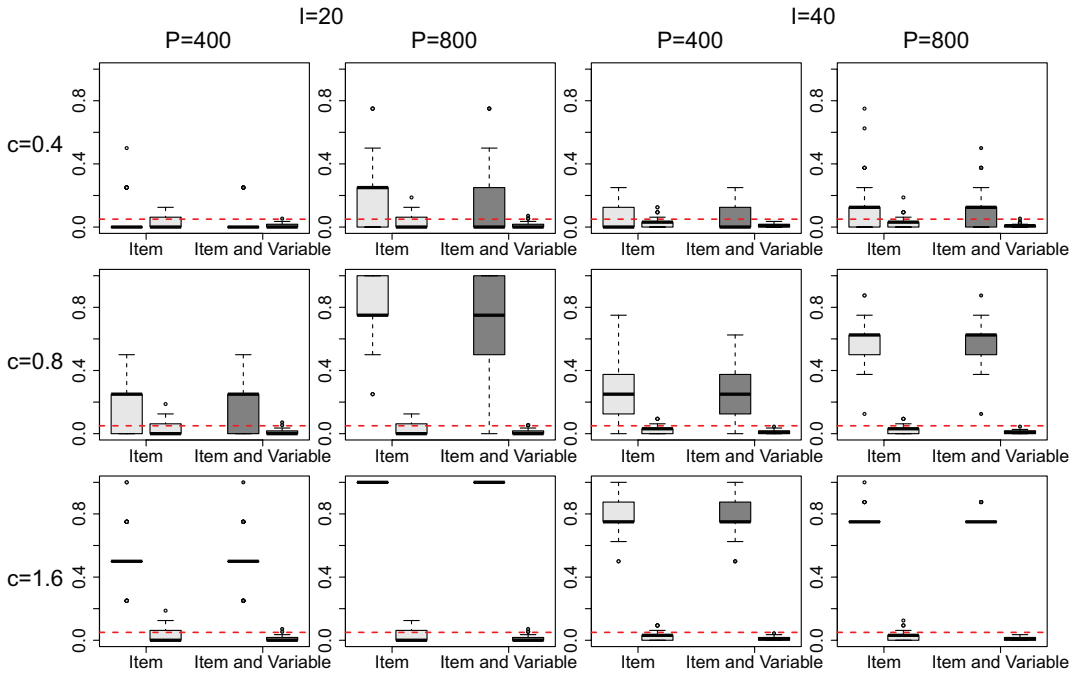

FIGURE 5. Boxplots of true positive rate and false positive rate at significance level $\alpha=.05$ (marked by dashed lines) in the simulation with three covariates and differential item functioning in $\mathrm{x}_{1}$. Results on item level are given in light gray and results for the combination of item and variable are given in dark gray. 
correlation $\rho=.6$ (not shown). It should be noted that in classical approaches for fixed groups, the simultaneous detection of DIF item and responsible variable is not investigated. If one considers more than one categorical variable, for example, gender and race, typically DIF induced by gender and race are investigated separately with significance levels fixed to the same value separately for the two investigations. However, it should be mentioned that in the extended logistic model, one could also investigate the effect of both variables by including both variables, and possibly an interaction term, in the linear predictor.

DIF in Two Covariates. In the following, we consider again the complex DIF structure considered in the illustrative example and use two DIF items. In Item 1, DIF is induced by $x_{1}$ and $x_{3}$ and determined by the step functions $b_{1, \bmod }=$ $b_{1}+c \cdot I\left(x_{3}>0\right)+c \cdot I\left(\left\{x_{3}>0\right\} \cap\left\{x_{1}=0\right\}\right)$, and in Item 2, DIF is induced by $x_{2}$ and $x_{3}$ and we use the step functions $b_{2, \bmod }=b_{2}+c \cdot I\left(x_{3}>0\right)+c$. $I\left(\left\{x_{3}>0\right\} \cap\left\{x_{2}=0\right\}\right)$. The strength of DIF again is determined by the additional parameter $c \in\{0.4,0.8,1.6\}$. By choosing these values for $c$, the differences between the individual groups remain the same as in the previous simulations.

In the same way as in Figure 5, the TPRs and FPRs of the 12 settings (with varying $I, P$, and $c$ ) based on 100 replications are given in Figure 6 . The TPRs on the item level (given in light gray) are very high for all settings with $c=0.8$ and $c=1.6$. Especially for the settings with $P=800$, the selection of items is quite perfect. However, for small DIF ( $c=0.4$, first row), the detection of responsible items remains quite challenging. It is also seen that the hit rates for the combination of item and variable (given in dark gray) are not much smaller than the hit rates for items. Since here DIF is generated by two variables, IFT cannot detect both variables in all the cases. However, the small FPRs show that the procedure does not tend to perform splits with regard to variables that are not responsible for DIF. If a significant effect is found, the corresponding split is always in the right variable.

\section{Investigation of NUDIF}

A strength of the logistic framework for DIF detection proposed by Swaminathan and Rogers (1990) is that it can be extended to detect NUDIF. We first consider the classical and extended approach and then the tree-based method.

\subsection{Logistic Regression for NUDIF}

Let us again first consider the comparison of multiple groups. To account for NUDIF, Model 1 has to be extended by group-specific slopes and has the form

$$
\eta_{p i}=\beta_{0 i}+S_{p} \beta_{i}+\gamma_{i g}+S_{p} \alpha_{i g},
$$




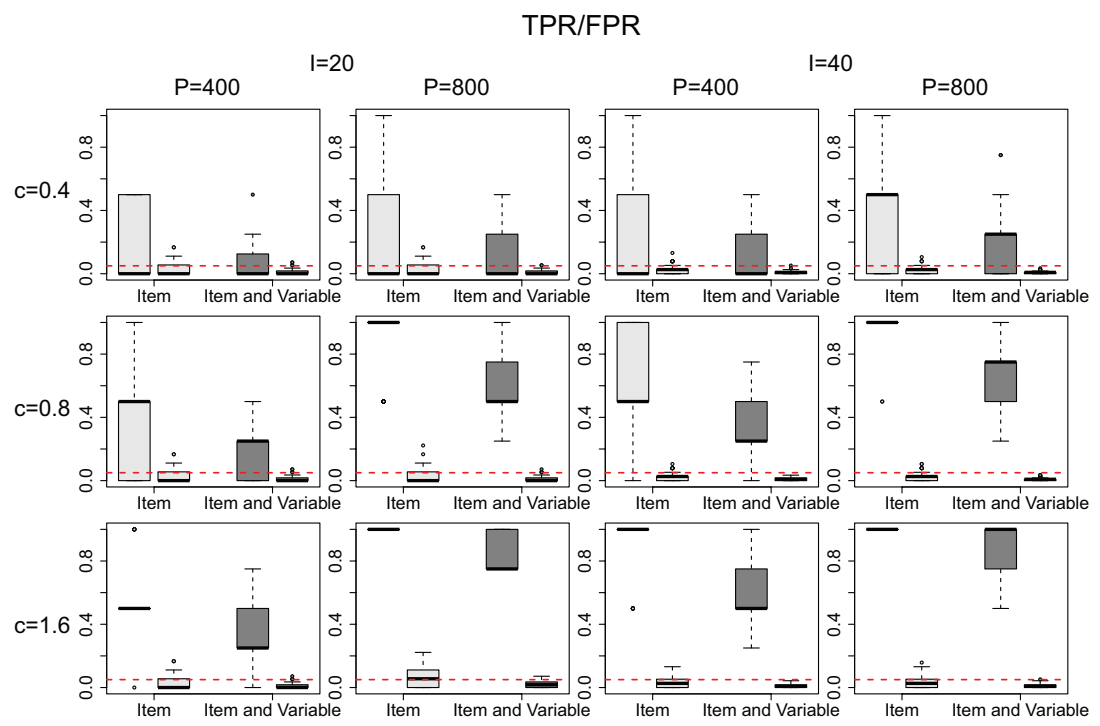

FIGURE 6. Boxplots of true positive rate and false positive rate at significance level $\alpha=.05$ (marked by dashed lines) in the simulation with three covariates and differential item functioning in 2 items and two covariates. Results on item level are given in light gray and results for the combination of item and variable are given in dark gray.

where $\alpha_{i g}$ are the additional group-specific slopes. The first group is chosen as reference group by setting $\gamma_{i 1}=\alpha_{i 1}=0$, see, for example, Magis et al. (2011). The model can be extended to account for NUDIF that is generated by a vector of covariates in a similar way as for UDIF. Then, one uses the model

$$
\eta_{p i}=\beta_{0 i}+S_{p} \beta_{i}+\mathbf{x}_{p}^{\top} \mathrm{g}_{i}+S_{p} \mathbf{x}_{p}^{\top} \boldsymbol{\alpha}_{i}
$$

which contains an interaction between the person characteristics $\mathbf{x}_{p}$ and the test score $S_{p}$. The new slope parameters in Model 8 are contained in $S_{p}\left(\beta_{i}+\mathbf{x}_{p}^{\top} \boldsymbol{\alpha}_{i}\right)$. Model 8 reduces to the logistic model used in Section 2 if $\boldsymbol{\alpha}_{i}=\mathbf{0}$. Thus, UDIF is present if $\boldsymbol{\gamma}_{i} \neq \mathbf{0}$ given $\boldsymbol{\alpha}_{i}=\mathbf{0}$. However, the item shows NUDIF if $\boldsymbol{\alpha}_{i} \neq \mathbf{0}$, whether $\boldsymbol{\gamma}_{i} \neq \mathbf{0}$ or not.

\subsection{Logistic Regression Trees for NUDIF}

When using the proposed tree-based model, NUDIF means that splits are not only admissible in the variables $x_{p 1}, \ldots, x_{p m}$ but also in the interaction terms 
$S_{p} x_{p 1}, \ldots, S_{p} x_{p m}$. A (first) split with regard to the interaction between the test score and the $j$ th variable yields the model with predictor

$$
\eta_{p i}=\beta_{0 i}+S_{p}\left[\alpha_{i l}^{[1]} I\left(x_{p j} \leq c_{j}\right)+\alpha_{i r}^{[1]} I\left(x_{p j}>c_{j}\right)\right],
$$

where the parameter $\alpha_{i l}^{[1]}$ denotes the slope in the left node $\left(x_{p j} \leq c_{j}\right)$ and $\alpha_{i r}^{[1]}$ denotes the slope in the right node $\left(x_{p j}>c_{j}\right)$.

\subsection{Test Strategies}

In the literature, different strategies were proposed how to test for the significance of DIF by means of Model 7 (see, e.g., Zumbo, 1999; Magis et al., 2011). We will use similar strategies when testing for DIF in the extended logistic regression Model 8 and the tree-based approach.

6.3.1. Testing for DIF. The first strategy is to test for both types of DIF effects simultaneously. The corresponding null hypothesis given in Model 7 is $H_{0}: \gamma_{i 2}=\ldots=\gamma_{i G}=\alpha_{i 2}=\ldots=\alpha_{i G}=0$. For Model 8, the corresponding null hypothesis is given by $H_{0}: \boldsymbol{\gamma}_{i}=\boldsymbol{\alpha}_{i}=0$. That means DIF is investigated by using a global test for the whole parameter vector $\left(\gamma_{i}, \alpha_{i}\right)$. DIF is considered as being present (in any form) if the test rejects the null hypothesis, meaning that at least one of the parameters $\gamma_{i j}, \alpha_{i j}, j=1, \ldots, m$, differs from zero.

For IFTs, the equivalent is that at least one split is performed in one of the components. When selecting the optimal split in each step of the algorithm, one has to consider all combinations of item, variable, split point, and component with regard to intercept and slope. The final model consists of one or two separate trees, one referring to the intercept and one referring to the slope. In general, the trees will be different but can also have the same structure. The resulting tree is given by

$$
\eta_{p i}=t r_{i}\left(\mathbf{x}_{p}\right)+t r_{i}\left(S_{p}, \mathbf{x}_{p}\right),
$$

where $\operatorname{tr}_{i}\left(\mathbf{x}_{p}\right)$ is the tree component containing subgroup-specific intercepts and $\operatorname{tr}_{i}\left(S_{p}, \mathbf{x}_{p}\right)$ is the tree component containing subgroup-specific slopes. In contrast to the tree in Model 5 for UDIF, now one has two possible trees. If there is only a significant effect in one of the two components, a constant $\operatorname{tr}_{i}\left(\mathbf{x}_{p}\right)=\beta_{0 i}$ or $\operatorname{tr}_{i}\left(S_{p}, \mathbf{x}_{p}\right)=S_{p} \beta_{i}$ is fitted in the other component.

In comparison to the classical and extended logistic method, the tree-based model has two advantages:

- The obtained tree(s) distinguishes between items with uniform and NUDIF. The trees themselves show which form of DIF is present. Thus, both types of DIF can be detected simultaneously within one fitting procedure.

- The obtained tree(s) identifies the variables that induce uniform and/or NUDIF. In particular, both types of DIF can be caused by different variables. 
6.3.2. Testing for NUDIF. A second strategy is to explicit test for NUDIF. Using the extended logistic Model 8, one investigates the null hypothesis $H_{0}: \boldsymbol{\alpha}_{i}=\mathbf{0}$ for each item. NUDIF is considered as being present if the hypothesis is rejected, meaning that at least one parameter $\alpha_{i j}$ differs from zero.

For IFT, the detection of NUDIF means that a significant split in the slope component is found. Consequently, during estimation, only the models with simultaneous splits in the intercepts and the slopes are considered as potential candidates. Therefore, one split in item $i$ with regard to variable $j$ corresponds to the model with predictor

$$
\eta_{p i}=\left[\gamma_{i l}^{[1]} I\left(x_{p j} \leq c_{j}\right)+\gamma_{i r}^{[1]} I\left(x_{p j}>c_{j}\right)\right]+S_{p}\left[\alpha_{i l}^{[1]} I\left(x_{p j} \leq c_{j}\right)+\alpha_{i r}^{[1]} I\left(x_{p j}>c_{j}\right)\right],
$$

which contains two intercepts $\left(\gamma_{i l}^{[1]}, \gamma_{i r}^{[1]}\right)$ and two slopes $\left(\alpha_{i l}^{[1]}, \alpha_{i r}^{[1]}\right)$ with respect to the same subgroups. To select the optimal split and to determine the splitting decision, one compares the likelihoods of Models 4 and 10. The procedure is continued in each step of the algorithm, considering all combinations of item, variable, and split point.

If NUDIF is present, the final model consists of two trees containing subgroup-specific intercepts and subgroup-specific slopes that are determined by the same splits.

For the different strategies, we will use the same terminology as Magis et al. (2011) in his investigation of the case in which DIF is induced by multiple groups:

- UDIF means testing for UDIF, $H_{0}: \boldsymbol{\gamma}_{i}=\mathbf{0}$, given Model 3 within the logistic regression approach. For trees, it refers to testing the corresponding splits.

- DIF means simultaneous tests for uniform and NUDIF, $H_{0}: \boldsymbol{\gamma}_{i}=\boldsymbol{\alpha}_{i}=\mathbf{0}$, given Model 8 for logistic regression. For trees, it refers to testing the corresponding splits for both types of DIF.

- NUDIF means tests for NUDIF, $H_{0}: \boldsymbol{\alpha}_{i}=\mathbf{0}$, given Model 8 for logistic regression. For trees, it refers to testing the corresponding splits.

\subsection{Illustrative Example}

As in Section 3, we consider data $Y_{p i}, p=1, \ldots, 800, i=1, \ldots, 20$, that are generated by a $2 \mathrm{PL}$ model with DIF. As before the item discrimination, parameters $a_{i}$ are first drawn from a uniform distribution. However, in order to simulate NUDIF, we do not generate data from the 2PL model but assume that the item discrimination parameters depend on covariates. The same strategy for generating NUDIF was also used by Rogers and Swaminathan (1993), Narayanan and Swaminathan (1996), or Jodoin and Gierl (2001).

Again, we consider 100 data sets with three covariates, two binary variables $x_{1}, x_{2} \sim B(1,0.5)$ and one standard normal distributed variable $x_{3} \sim N(0,1)$. We 
TABLE 3.

Modified Values of Item Discrimination and Item Difficulty Parameters in the Illustrative Example With Nonuniform DIF.

\begin{tabular}{llcc}
\hline Item & Nonuniform DIF & Item & Uniform DIF \\
\hline 1 & $a_{1, \bmod }=a_{1}+0.6 \cdot I\left(x_{1}=1\right)$ & 3 & $b_{3, \bmod }=b_{3}+0.8 \cdot I\left(x_{1}=1\right)$ \\
2 & $a_{2, \text { mod }}=a_{2}+0.6 \cdot I\left(x_{2}=0\right)$ & 4 & $b_{4, \text { mod }}=b_{4}+0.8 \cdot I\left(x_{2}=0\right)$ \\
\hline
\end{tabular}

Note. DIF $=$ differential item functioning.

simulate data where 2 of the 20 items show NUDIF and 2 of the 20 items only show UDIF. The modified values of the discrimination and difficulty parameters are determined by step function given in Table 3. In Items 1 and 3, DIF is induced by $x_{1}$; and in Items 2 and 4, DIF is induced by $x_{2}$. Hence, in all four cases, two groups have to be distinguished. The resulting ICCs of the 2 items with NUDIF (Items 1 and 2) are given in Figure 7 separately for the two groups. It can be seen from the curves that the item locations are equal for both groups, but the item discriminations (as it was simulated) differ between the groups. When fitting IFT, the NUDIF structure is detected correctly if there is one split in the slope component of the model of Item 1 in $x_{1}$ and Item 2 in $x_{2}$.

$D I F$. Figure 8 shows one exemplary estimation result obtained by IFT when testing for both types of DIF simultaneously. In this example, Items 1-4 are correctly identified as DIF items. All items are split once yielding trees with two terminal nodes, respectively. Items 1 and 2 (upper panel) are split with regard to the slopes indicating NUDIF. In Item 1, the (simulated) item discrimination is higher for $\left\{x_{1}=1\right\}$, yielding a higher slope for the corresponding subgroup $\left(\hat{\alpha}_{1, x_{1}=1}=0.328\right)$. Whereas, in Item 2 , the item discrimination is larger for $\left\{x_{2}=0\right\}$, which results in a larger slope for this subgroup $\left(\hat{\alpha}_{2, x_{2}=0}=0.298\right)$. In Items 3 and 4 (lower panel), one split is performed with regard to the intercepts, indicating UDIF. The results are also in line with the true simulated effects. The model provides an identification of DIF items together with the responsible covariates and a classification by type of DIF.

NUDIF. When using IFT, which explicitly tests for NUDIF, only Items 1 and 2, which were simulated as NUDIF items, are detected. The corresponding trees are given in Figure 9. The subgroup-specific slopes (left panel) are defined by the same splits as in the DIF framework considered previously. Due to the construction of the model, the estimated coefficients $\alpha_{i 1}, \alpha_{i 2}, i=1,2$, however, differ slightly. If splits are significant, the same splits are performed in the intercepts yielding trees with subgroup-specific intercepts. Since they are not of main interest, they are displayed a little smaller (right panel of Figure 9). 

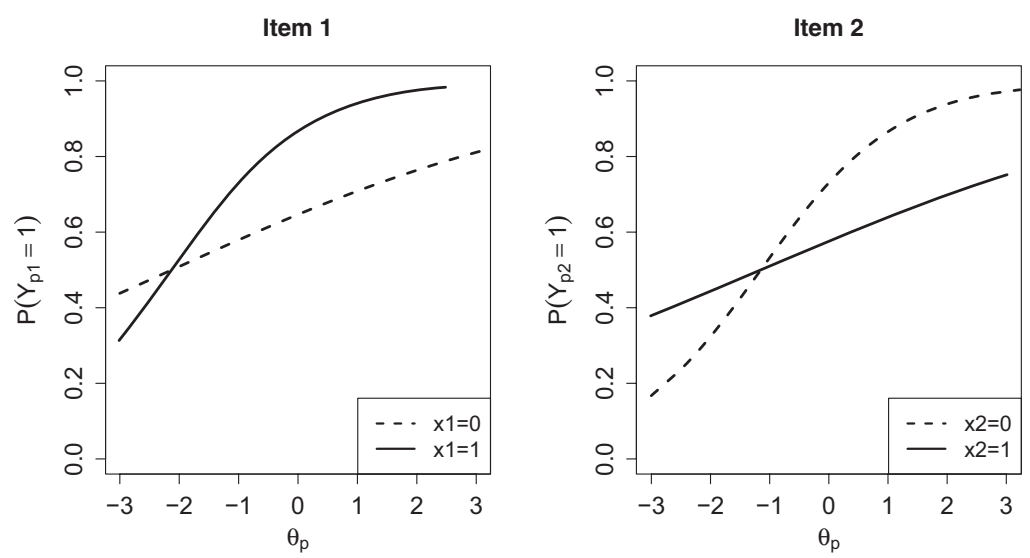

FIGURE 7. Item characteristic curves of Items 1 and 2 for the illustrative example with nonuniform differential item functioning.

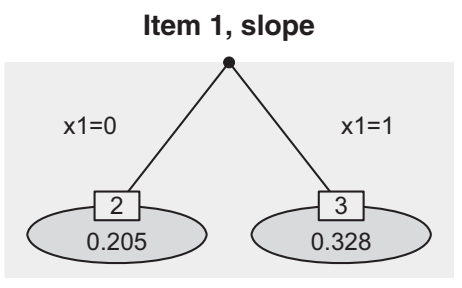

Item 3, intercept

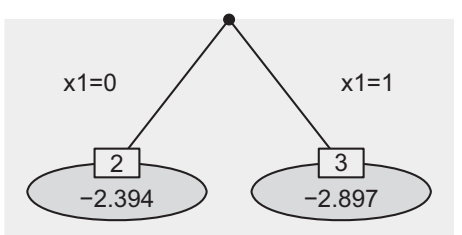

Item 2, slope

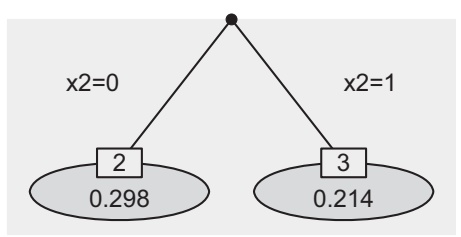

Item 4, intercept

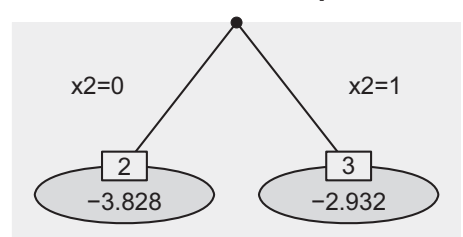

FIGURE 8. Estimated trees for the illustrative example with nonuniform differential item functioning (DIF), testing for both types of DIF. Estimated coefficients $\alpha_{i \ell}$ (upper) and $\gamma_{i \ell}$ (lower) are given in each leaf of the trees.

\subsection{Simulations}

In the following, we briefly illustrate the properties of the models for the DIF and NUDIF framework by means of a small simulation. The structure of the simulated data sets we consider here is the same as in Section 5. We limit the discussion to the comparison of two groups defined by one binary covariate $x \in\{0,1\}$. According to Model 6, NUDIF is present if the item discriminations 

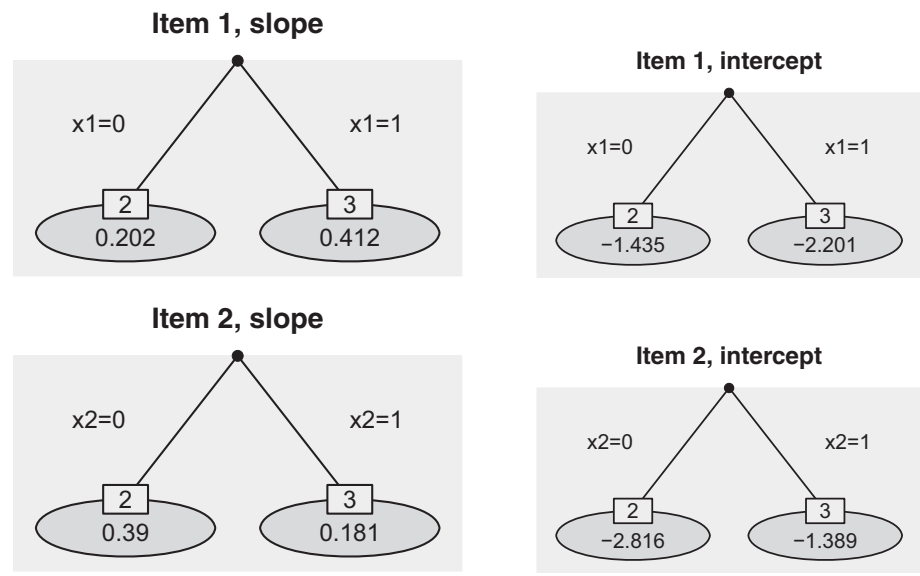

FIGURE 9. Estimated trees for the illustrative example with nonuniform differential item functioning (NUDIF), testing for NUDIF. Estimated coefficients $\alpha_{i \ell}$ (left) and $\gamma_{i \ell}$ (right) are given in each leaf of the trees.

$a_{i}$ differ between the two groups. The difference in item discriminations is simulated by the equation $a_{i, \bmod }=a_{i}+c \cdot I(x=0)$ for one half of DIF items and by the equation $a_{i, \bmod }=a_{i}+c \cdot I(x=1)$ for the other half of DIF items, with constant $c \in\{0.3,0.6\}$. From our experience, the values 0.3 and 0.6 represent medium DIF effect sizes. Boxplots of true positive and FPRs on the item level for the setting with $P=800, I=20$, and $20 \%$ DIF obtained by IFT (left of each panel) and the classical logistic model (right of each panel) are given in Figure 10. The results when testing for both types of DIF are shown in the left panel and the results when testing for NUDIF are shown in the right panel. Within the DIF framework, the classical logistic model outperforms the proposed treebased approach. The average hit rate in the setting with $c=0.6$ (lower left) is 0.66 for logistic, but only 0.43 for IFT. This was to be expected because the test on the whole parameter vector $\left(\gamma_{i}, \alpha_{i}\right)$ obviously has a stronger power than the tests on single splits. However, in the NUDIF framework, the two methods almost yield the same results. The average hit rate in the settings with $c=0.6$ (lower right) for both models is 0.44 . Due to the construction of the models, the main difference in the case of two groups is the use of different test statistics to obtain a decision. As we already illustrated for UDIF, our proposed IFT approach can also be used to detect NUDIF without loss of efficiency. The findings presented here can be confirmed by the results of all other settings considered in our simulation.

\section{Empirical Applications}

Finally, we will illustrate and compare the proposed approaches on real data examples. 


\section{TPR/FPR}
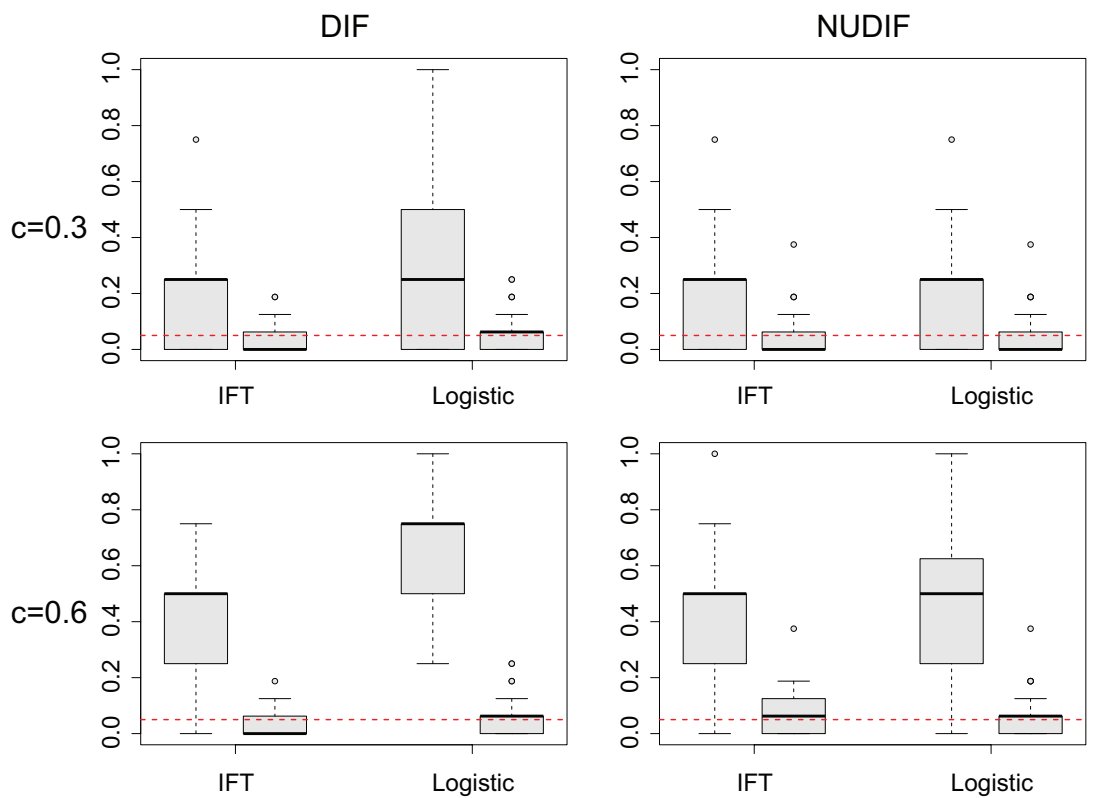

FIGURE 10. Boxplots of true positive rate and false positive rate for the simulation with nonuniform differential item functioning (NUDIF) and one binary predictor ( $\mathrm{P}=800$, $\mathrm{I}=20,20 \%$ DIF), testing for both types of DIF (left) and testing for NUDIF (right).

\subsection{Intelligence-Structure-Test 2000 R (I-S-T 2000 R)}

We use data from the I-S-T 2000 R (Testzentrale Göttingen Göttingen, www.testzentrale.de). The test was developed by Amthauer, Brocke, Liepmann, and Beauducel (2001) and Beauducel, Liepmann, Horn, and Brocke (2010) and is a revised version of its predecessors I-S-T 70 (Amthauer, Brocke, Liepmann, \& Beauducel, 1973) and I-S-T 2000 (Amthauer, Brocke, Liepmann, \& Beauducel, 1999). The available study was conducted at the Phillips University in Marburg (Bühner, Ziegler, Krumm, \& Schmidt-Atzert, 2006). There were 273 participants from 40 different subject areas. The second module of the test contains 20 items (Items 21-40) in which analogies play the major role. There are three predefined terms with a certain relation between the first two. This relationship needs to be recognized to find the fourth term. From five possible answers, the respondent is asked to choose the term that relates to the third term, as the second term relates to the first term. One example is

\section{dark : bright wet : ?}
(a) rain,
(b) day,
(c) moist,
(d) wind,
(e) dry. 
TABLE 4.

Summary Statistics of the Test Score of the Second Module (Items 21-40) of the I-S-T 2000 $R$ and the Two Considered Covariates.

\begin{tabular}{lrccccc}
\hline & \multicolumn{6}{c}{ Summary Statistics } \\
\cline { 2 - 7 } Variable & $x_{\min }$ & $x_{0.25}$ & $x_{\text {med }}$ & $\bar{x}$ & $x_{0.75}$ & $x_{\max }$ \\
\hline Test score & 6 & 12 & 14 & 13.87 & 16 & 19 \\
Age & 18 & 20 & 22 & 22.88 & 24 & 39 \\
Gender & & Male: 97 & & & Female: 176 & \\
\hline
\end{tabular}

Note. I-S-T $2000 \mathrm{R}=$ Intelligence-Structure-Test $2000 \mathrm{R}$.
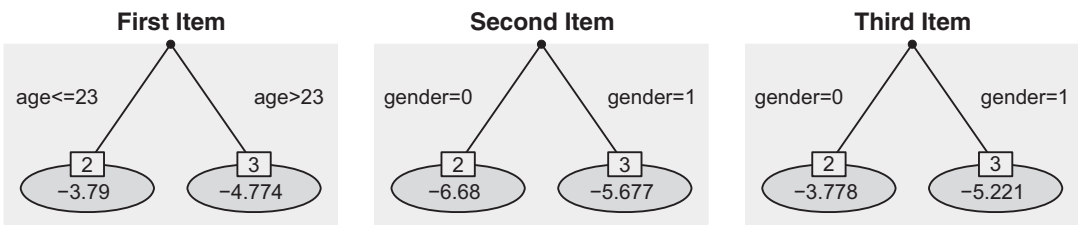

FIGURE 11. Trees of the three detected differential item functioning (DIF) items of the second module of the Intelligence-Structure-Test $2000 \mathrm{R}$ using the model for uniform DIF. Estimated intercepts $\gamma_{i \ell}$ are given in each leaf of the trees.

Therefore, one has to select that alternative that relates to wet as bright relates to dark.

For the investigation of DIF in these items, we incorporate the covariates gender (male: 0 , female: 1) and age. The summary statistics of the resulting test scores of Items 21-40 and the two covariates are given in Table 4.

When using IFT for UDIF, 3 of the 20 items showed DIF. The algorithm performs only three splits before stopping, and therefore, each item is split only once. All permutation tests were based on 1,000 permutations at local significance level $.05 / 2$.

The estimated trees for 3 items detected as DIF items are given in Figure 11. It is seen that both covariates gender and age seem to induce DIF because both are used for splitting at least once. The second and third item show DIF induced by gender, whereas the first item shows DIF induced by age. According to the estimated coefficients, the second item is easier for females (gender $=1)$, the third item is easier for males (gender $=0$ ), and the first item is easier for all students who are rather young (age $\leq 23$ ).

An overview of the detected DIF items obtained by the six strategies discussed in this article is given in Table 5. When using IFT which tests for both types of DIF, one obtains very similar results. As in the UDIF framework, the first, second, and third items are also identified as DIF items with the same variables 
TABLE 5.

Comparison of Detected DIF Items of the I-S-T 2000 R Using IFT and the Extended Logistic Approach for Uniform and Nonuniform DIF.

\begin{tabular}{|c|c|c|c|c|c|c|c|}
\hline \multirow[b]{2}{*}{ Item } & \multicolumn{4}{|c|}{ IFT } & \multicolumn{3}{|c|}{ Extended Logistic } \\
\hline & UDIF & DIF & & NUDIF & UDIF & DIF & NUDIF \\
\hline First & $x$ & $x$ & (u) & & $x$ & $x$ & \\
\hline Second & $x$ & $x$ & (u) & & $x$ & $x$ & \\
\hline Third & $x$ & $x$ & (non) & & $x$ & $x$ & \\
\hline Fourth & & & & & $x$ & & \\
\hline Fifth & & & & & $\times$ & & \\
\hline
\end{tabular}

Note. FPR $=$ false positive rate; DIF $=$ differential item functioning; IFT $=$ item-focused trees; UDIF $=$ uniform DIF; NUDIF = nonuniform DIF; I-S-T $2000 \mathrm{R}=$ Intelligence-Structure-Test $2000 \mathrm{R}$.

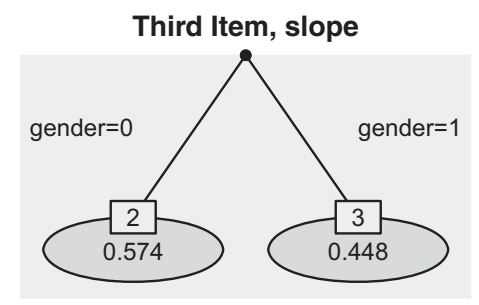

FIGURE 12. Tree of the third detected differential item functioning (DIF) item of the second module of the Intelligence-Structure-Test $2000 \mathrm{R}$ using the model for both types of $D I F$. Estimated slopes $\alpha_{i \ell}$ are given in each leaf of the trees.

that induce DIF. The estimated models for the first and second items are even identical. A difference occurs for the third item, where the split in gender is not performed in the intercept but in the slope component. The model gives the estimated intercept $\beta_{0, \text { third }}=-4.993$. The resulting tree of slopes $\alpha_{i l}$ is given in Figure 12. The estimated coefficients again mean that the item favors males (gender $=0$ ), but the difference slightly increases for participants with a higher test score. Interestingly, the splits in the intercept (UDIF, Figure 11) and in the slope (DIF, Figure 12) result in very similar estimated probabilities. As a consequence, it is not surprising that the third item is not detected by the model within the NUDIF framework.

The evaluation of the data set by the extended logistic Model 3 for UDIF yields 5 DIF items (fourth column in Table 5). Based on the results in the simulations, it seems that the fourth and fifth item might be falsely identified as items with UDIF. Concerning the identification of items, the results within the DIF and NUDIF framework are equal to those of IFT. However, when testing NUDIF for the third item, one obtains the $p$ value .052 indicating an almost 
TABLE 6.

Overview on Estimated Effect Sizes of the I-S-T 2000 R Using IFT and the Extended Logistic Approach for Uniform DIF.

\begin{tabular}{|c|c|c|c|c|}
\hline \multicolumn{3}{|c|}{ Item-Focused Trees } & \multicolumn{2}{|c|}{ Extended Logistic } \\
\hline Item & Age & Gender & Age & Gender \\
\hline First & .984 & $\times$ & $-.943(.152)$ & $-.026(.154)$ \\
\hline Second & $\times$ & 1.002 & $.091(.165)$ & $.507(.174)$ \\
\hline Third & $x$ & 1.443 & $.485(.212)$ & $-.583(.225)$ \\
\hline Fourth & $x$ & $x$ & $.175(.200)$ & $-.455(.237)$ \\
\hline Fifth & $x$ & $\times$ & $.088(.133)$ & $.367(.138)$ \\
\hline
\end{tabular}

Note. For IFT, the differences of the effects in the nodes are given, and for the logistic approach, the estimates and standard errors are given. DIF $=$ differential item functioning; IFT $=$ item-focused trees; I-S-T $2000 \mathrm{R}=$ Intelligence-Structure-Test $2000 \mathrm{R}$.

significant effect. Table 6 shows a detailed overview of the estimated DIF effect sizes when using the two approaches for UDIF. For IFT (left columns), the given values correspond to the (norm of the) differences of the estimated values in the nodes of the trees in Figure 11. For the third item, one observes the difference 1.443 , which is quite large. The extended logistic approach does not explicitly provide information about the variables that are responsible for DIF, but the estimates and corresponding standard errors given in Table 6 indicate which ones might be relevant.

It is noteworthy that in summary, the test seems not to be strongly affected by DIF. From the 20 items that use analogies, only 3 are suspect of DIF and the effects are not overly strong. This was to be expected of a carefully designed test.

\subsection{California Testing Bureau (CTB) Science Data}

In a second application, we consider a data set from CTB-McGraw Hill. For a description of the original data, see also De Boeck and Wilson (2004). The data include the results of 1,500 Grade 8 students from 35 schools. The students had to respond to 76 items, measuring different objectives and subskills related to mathematics and science. In our investigation, we restrict to the 25 multiplechoice items from subject area science.

To test for DIF in these items, we incorporate the three covariates gender (male: 0, female: 1), type of the school (1: catholic, 2: private, 3: public), and size of the school (number of students in hundreds). The summary statistics of the test scores for the 25 items and the three covariates are given in Table 7.

When fitting IFT for UDIF, 14 of the 25 items are identified as DIF items. Altogether, the algorithm performs 27 splits until further splits are no longer 
Berger and Tutz

TABLE 7.

Summary Statistics of the Test Score of the 25 Multiple-Choice Items From Subject Area Science of the CTB Data and the Three Considered Covariates.

\begin{tabular}{lcccccc}
\hline \multirow{2}{*}{ Variable } & \multicolumn{6}{c}{ Summary Statistics } \\
\cline { 2 - 7 } & $x_{\min }$ & $x_{0.25}$ & $x_{\text {med }}$ & $\bar{x}$ & $x_{0.75}$ & $x_{\max }$ \\
\hline Test score & 7 & 14 & 16 & 16.01 & 18 & 23 \\
Size & 100 & 500 & 900 & 868.3 & 1,300 & 1,600 \\
Type & Catholic: 105 & Private: 84 & Public: 1,311 \\
Gender & & Male: 761 & & \multicolumn{3}{c}{ Female: 739} \\
\hline
\end{tabular}

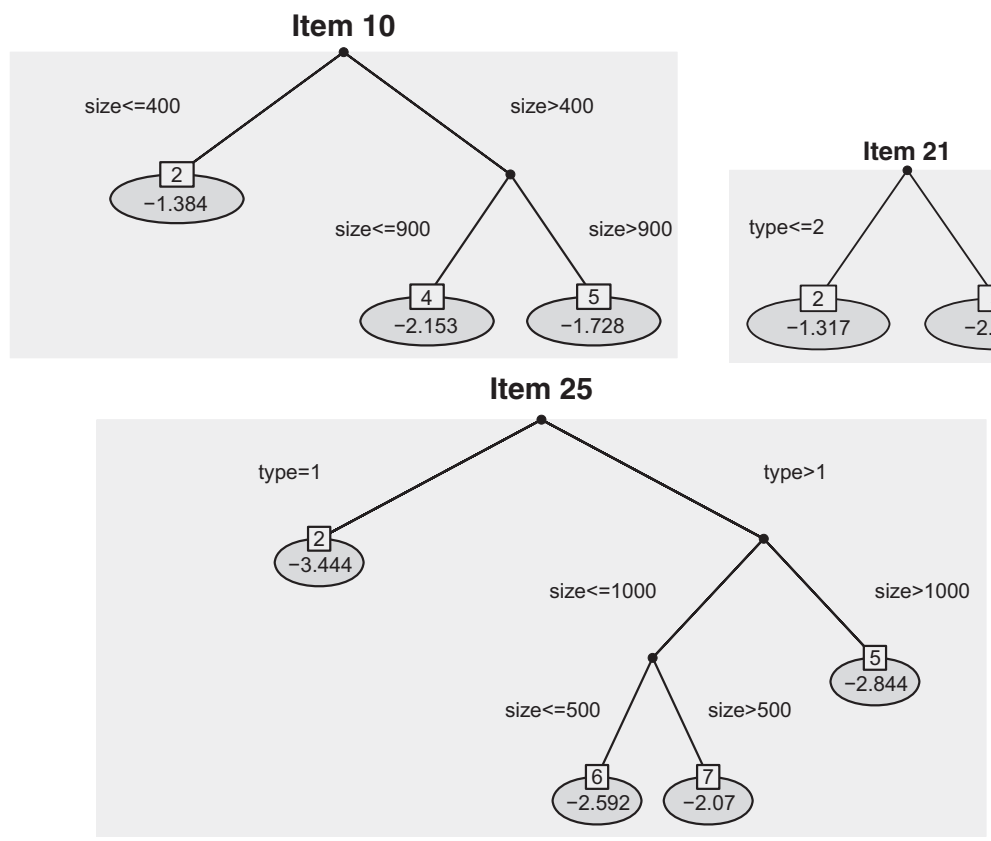

FIGURE 13. Trees of Items 10, 21, and 25 of the CTB data using the model for uniform differential item functioning. Estimated intercepts $\gamma_{i \ell}$ are given in each leaf of the trees.

significant. With three covariates, each permutation test is performed at local significance level $.05 / 3$. The $p$ value in the 28th iteration was .02 and thus not significant on level $.01 \overline{6}$. All splits refer to covariates type and size, whereas no significant splits were found for variable gender. There does not seem to be any difference between males and females. 
TABLE 8.

Comparison of Detected DIF Items of the CTB Data Using IFT and the Extended Logistic Approach for Uniform and Nonuniform DIF.

\begin{tabular}{|c|c|c|c|c|c|c|c|}
\hline \multirow[b]{2}{*}{ Item } & \multicolumn{4}{|c|}{ IFT } & \multicolumn{3}{|c|}{ Extended Logistic } \\
\hline & UDIF & DIF & & NUDIF & UDIF & DIF & NUDIF \\
\hline 21 & $x$ & $x$ & (non) & $x$ & $x$ & $x$ & $x$ \\
\hline 3 & $x$ & $x$ & (u) & & $x$ & $x$ & \\
\hline 4 & $\times$ & $\times$ & (u) & & $\times$ & $\times$ & \\
\hline 8 & $x$ & $x$ & (u) & & $x$ & $x$ & \\
\hline 9 & $x$ & $x$ & (u) & & $x$ & $x$ & \\
\hline 14 & $x$ & $x$ & (non) & & $x$ & $x$ & \\
\hline 16 & $\times$ & $x$ & (non) & & $x$ & $x$ & \\
\hline 25 & $\times$ & $x$ & (u) & & $x$ & $x$ & \\
\hline 11 & & & & & $\times$ & $\times$ & $\times$ \\
\hline 13 & & & & $x$ & & $x$ & $\times$ \\
\hline 19 & $\times$ & $x$ & (u) & & $x$ & & \\
\hline 5 & $x$ & $x$ & (u) & & & & \\
\hline 10 & $x$ & $x$ & (u) & & & & \\
\hline 24 & & & & & $x$ & $x$ & \\
\hline 1 & & & & & & & $x$ \\
\hline 6 & $\times$ & & & & & & \\
\hline 15 & $\times$ & & & & & & \\
\hline 17 & $\times$ & & & & & & \\
\hline
\end{tabular}

Note. DIF $=$ differential item functioning; IFT $=$ item-focused trees; UDIF $=$ uniform DIF NUDIF $=$ nonuniform DIF.

The trees for three selected items are given in Figure 13. In Item 10, DIF is induced by size and one has to distinguish between three subgroups. The item is easiest for students in small schools (size $\leq 400$ ) but most difficult for students in medium-sized schools $(400<$ size $\leq 900)$. Item 21 is easier for students in a catholic or private school (type $\leq 2$ ) compared to students in public schools (type $=3$ ). An interesting partition is received for Item 25 . For all students in a catholic school (type =1), the question is very difficult. By contrast, the question is easier for all students in a private or public school (type $>1$ ) in particular for those in medium-sized schools $(500<$ size $\leq 1,000)$.

To obtain DIF effect sizes, we computed the maximal difference of estimated effects between any two nodes for each tree. The obtained values vary over a wide range from 0.458 to 2.985 . This also confirms that large DIF effects such as 1.6 might occur in real data sets.

An overview of the detected DIF items by the six evaluated models is given in Table 8. It shows only items that were found to be DIF items by at least one of the models. Within the DIF framework (second column), 11 DIF items are identified. 


\section{Berger and Tutz}

These are the same items as with the restricted model for UDIF discussed above but without Items 6,15 , and 17 . Unlike above, there are 3 items that are classified as NUDIF items by the more general model. Here, for example, in Item 21, the split regarding the type of school is not performed in the intercept but in the slope component. According to the model testing for NUDIF (third column), the 2 items, 13 and 21, carry NUDIF. In contrast to Item 13, Item 21 is also detected within the UDIF and DIF framework.

The comparison to the extended logistic approach shows a strong overlap. Within the UDIF framework (first and fourth column), there is an agreement in 9 items. In the DIF framework, this is the case for 8 items. However, it should again be mentioned that the extended logistic approach within the DIF framework does not distinguish between uniform and NUDIF. When testing for NUDIF (sixth column), one obtains four significant results. In contrast to Items 1 and 11 , Items 13 and 21 are also found by IFT. In total, Item 21 is the only item that shows DIF according to all six models, and 4 items are only identified as DIF items by one of the six models.

\section{Concluding Remarks}

The proposed recursive partitioning approach, in short IFT, is an extension of the basic logistic regression model for the detection of uniform and NUDIF. In contrast to the classical approach, IFT allows to incorporate several covariates on different scales, including ordinal and continuous covariates, that potentially induce DIF. The method leads to simultaneous selection of items and (interactions of) variables that cause DIF. The result typically is a small tree for each DIF item, and therefore the DIF structure is easy accessible.

The results of the simulations including uniform as well as NUDIF show that IFT has the same performance as the classical approach in the simple case of two groups but also works quite well in more complex settings with various covariates. Nevertheless, it should be noted that in the latter case, the method is conservative and does not exploit the significance level fully. The applications demonstrate the flexibility and interpretability of IFT, also compared to the extended logistic model that tests DIF by a vector of covariates. In particular, within the framework that tests for both types of DIF, the obtained trees show which type of DIF is present. The results shown in the article were obtained by an $R$ program that was written by one of the authors and is available in the $R$ add-on package DIFtree (Berger, 2016; $R$ Core Team, 2015).

\section{Acknowledgement}

We gratefully acknowledge support from Deutsche Forschungsgemeinschaft (DFG Project TU 62/8-1: Differential Item Functioning in Item Response Models). 


\section{Declaration of Conflicting Interests}

The author(s) declared no potential conflicts of interest with respect to the research, authorship, and/or publication of this article.

\section{Funding}

The author(s) received no financial support for the research, authorship, and/or publication of this article.

\section{References}

Amthauer, R., Brocke, B., Liepmann, D., \& Beauducel, A. (1973). Intelligenz-StrukturTest (IST 70). Göttingen, Germany: Hogrefe.

Amthauer, R., Brocke, B., Liepmann, D., \& Beauducel, A. (1999). Intelligenz-StrukturTest 2000 (I-S-T 2000). Göttingen, Germany: Hogrefe.

Amthauer, R., Brocke, B., Liepmann, D., \& Beauducel, A. (2001). Intelligenz-StrukturTest 2000 R (I-S-T 2000 R)-Handanweisung. Göttingen, Germany: Hogrefe.

Beauducel, A., Liepmann, D., Horn, S., \& Brocke, B. (2010). Intelligence structure testEnglish version of the Intelligenz-Struktur-Test 2000 R (I-S-T 2000 R). Göttingen, Germany: Hogrefe.

Berger, M. (2016). DIFtree: Item focused trees for the identification of items in differential item functioning. $R$ package version 2.0.2. Retrieved from http://CRAN.R-pro ject.org/package $=$ DIFtree

Bühner, M., Ziegler, M., Krumm, S., \& Schmidt-Atzert, L. (2006). Ist der I-S-T 2000 R Rasch-skalierbar? Diagnostica, 52, 119-130.

Breiman, L., Friedman, J. H., Olshen, R. A., \& Stone, J. C. (1984). Classification and regression trees. Monterey, CA: Wadsworth.

De Boeck, P., \& Wilson, M. (2004). Explanatory item response models: A generalized linear and nonlinear approach. New York, NY: Springer Verlag.

Hastie, T., Tibshirani, R., \& Friedman, J. H. (2009). The elements of statistical learning (2nd ed.). New York, NY: Springer-Verlag.

Holland, P. W., \& Thayer, D. T. (1988). Differential item performance and the MantelHaenszel procedure. In H. Wainer \& H. I. Braun (Eds.), Test validity (pp. 129-145). Hillsdale, NJ: Erlbaum.

Holland, W., \& Wainer, H. (1993). Differential item functioning. Hillsdale, NJ: Lawrence Erlbaum.

Hothorn, T., Hornik, K., \& Zeileis, A. (2006). Unbiased recursive partitioning: A conditional inference framework. Journal of Computational and Graphical Statistics, 15, 651-674.

Hothorn, T., \& Lausen, B. (2003). On the exact distribution of maximally selected rank statistics. Computational Statistics and Data Analysis, 43, 121-137.

Jodoin, M., \& Gierl, M. (2001). Evaluating type I error and power rates using an effect size measure with the logistic regression procedure for DIF detection. Applied Measurement in Education, 14, 329-349.

Lord, F. M. (1980). Applications of item response theory to practical testing problems. New York, NY: Routledge. 
Magis, D., Bèland, S., Tuerlinckx, F., \& Boeck, P. (2010). A general framework and an R package for the detection of dichotomous differential item functioning. Behavior Research Methods, 42, 847-862.

Magis, D., Raîche, G., Béland, S., \& Gérard, P. (2011). A generalized logistic regression procedure to detect differential item functioning among multiple groups. International Journal of Testing, 11, 365-386.

Magis, D., Tuerlinckx, F., \& De Boeck, P. (2015). Detection of differential item functioning using the lasso approach. Journal of Educational and Behavioral Statistics, 40, $111-135$.

Millsap, R., \& Everson, H. (1993). Methodology review: Statistical approaches for assessing measurement bias. Applied Psychological Measurement, 17, 297-334.

Narayanan, P., \& Swaminathan, H. (1996). Identification of items that show nonuniform DIF. Applied Psychological Measurement, 20, 257-274.

Osterlind, S. J., \& Everson, H. T. (2009). Differential item functioning, Volume 161. Thousand Oaks, CA: SAGE.

Raju, N. S. (1988). The area between two item characteristic curves. Psychometrika, 53, 495-502.

$R$ Core Team. (2015). $R$ : A language and environment for statistical computing, Version 3.2.2. Vienna, Austria: Foundation for Statistical Computing. Retrieved from https:// www.R-project.org/

Rogers, H. J. (2005). Differential item functioning. Encyclopedia of Statistics in Behavioral Science. Colchester, UK: John Wiley \& Sons.

Rogers, H., \& Swaminathan, H. (1993). A comparison of logistic regression and MantelHaenszel procedures for detecting differential item functioning. Applied Psychological Measurement, 17, 105-116.

Schauberger, G., \& Tutz, G. (2015). Detection of differential item functioning in Rasch models by boosting techniques. British Journal of Mathematical and Statistical Psychology. doi:10.1111/bmsp. 12060

Shih, Y.-S. (2004). A note on split selection bias in classification trees. Computational Statistics and Data Analysis, 45, 457-466.

Shih, Y.-S., \& Tsai, H. (2004). Variable selection bias in regression trees with constant fits. Computational Statistics and Data Analysis, 45, 595-607.

Strobl, C., Boulesteix, A.-L., \& Augustin, T. (2007). Unbiased split selection for classification trees based on the Gini index. Computational Statistics \& Data Analysis, 52, 483-501.

Strobl, C., Kopf, J., \& Zeileis, A. (2015). Rasch trees: A new method for detecting differential item functioning in the Rasch model. Psychometrika, 80, 289-316.

Strobl, C., Malley, J., \& Tutz, G. (2009). An introduction to recursive partitioning: Rationale, application and characteristics of classification and regression trees, bagging and random forests. Psychological Methods, 14, 323-348.

Swaminathan, H., \& Rogers, H. J. (1990). Detecting differential item functioning using logistic regression procedures. Journal of Educational Measurement, 27, 361-370.

Tutz, G., \& Berger, M. (2015). Item focussed trees for the identification of items in differential item functioning. Psychometrika. doi:10.1007/s11336-015-9488-3 
Tutz, G., \& Schauberger, G. (2015). A penalty approach to differential item functioning in Rasch models. Psychometrika, 80, 21-43.

Zumbo, B. (1999). A handbook on the theory and methods of differential item functioning (dif). Ottawa, Canada: National Defense Headquarters.

\section{Authors}

MORITZ BERGER is a PhD student at the Department of Statistics, Ludwig-MaximiliansUniversität München, Germany; e-mail: moritz.berger@stat.unimuenchen.de. His research interests are recursive partitioning methods, differential item functioning, and categorical response models.

GERHARD TUTZ is a full professor at the Department of Statistics, Ludwig-MaximiliansUniversität München, Germany; e-mail: tutz@stat.uni-muenchen.de. His research interests are categorical data, mixture models, survival models and latent trait models.

Manuscript received January 8, 2016 First revision received March 17, 2016

Second revision received May 18, 2016

Accepted June 10, 2016 